\title{
Modeling of conductive particle motion in viscous medium affected by an electric field considering particle-electrode interactions and microdischarge phenomenon
}

\author{
Ghiyam Eslami, ${ }^{1, a)}$ Esmaeil Esmaeilzadeh, ${ }^{1, b)}$ and Alberto T. Pérez ${ }^{2, c)}$ \\ ${ }^{1}$ Department of Mechanical Engineering, University of Tabriz, Tabriz, Iran \\ ${ }^{2}$ Departamento de Electrónica y Electromagnetismo, Facultad de Física, \\ Universidad de Sevilla, Avenida Reina Mercedes s/n, 41012 Sevilla, Spain
}

(Received 11 February 2016; accepted 28 September 2016; published online 17 October 2016)

\begin{abstract}
Up and down motion of a spherical conductive particle in dielectric viscous fluid driven by a DC electric field between two parallel electrodes was investigated. A nonlinear differential equation, governing the particle dynamics, was derived, based on Newton's second law of mechanics, and solved numerically. All the pertaining dimensionless groups were extracted. In contrast to similar previous works, hydrodynamic interaction between the particle and the electrodes, as well as image electric forces, has been taken into account. Furthermore, the influence of the microdischarge produced between the electrodes and the approaching particle on the particle dynamics has been included in the model. The model results were compared with experimental data available in the literature, as well as with some additional experimental data obtained through the present study showing very good agreement. The results indicate that the wall hydrodynamic effect and the dielectric liquid ionic conductivity are very dominant factors determining the particle trajectory. A lower bound is derived for the charge transferred to the particle while rebounding from an electrode. It is found that the time and length scales of the post-microdischarge motion of the particle can be as small as microsecond and micrometer, respectively. The model is able to predict the so called settling/dwelling time phenomenon for the first time. Published by AIP Publishing. [http://dx.doi.org/10.1063/1.4964683]
\end{abstract}

\section{INTRODUCTION}

Small conductive particles exposed to a DC electric field are encountered in various technical and industrial situations, such as electrostatic technologies, high voltage power systems, printing equipment, surface coating, separation of mixed granular materials, ${ }^{1}$ and biological cell manipulation. A deep understanding of the particles dynamics is required for designing and maintaining such systems. Also, experimental progresses during past few years in the areas of a microelectromechanical systems (MEMS), nano-science, and microfluidics have intensified the interest in the control of motion and manipulation of particles by electrical forces. ${ }^{2}$ Accurate prediction and control of conductive particles motion would improve the performance and efficiency of high voltage technologies.

One of the common configurations involving tiny conductive particles under influence of a DC electrical field refers to the recycling system of plastic waste. ${ }^{3}$ In this configuration, an electric force is applied to remove conductive impurities, like carbon particles, from liquefied plastic., ${ }^{4,5}$ Also, it has been shown that the motion of a conductive particle in a typical parallel electrode system can be used for measuring the viscosity of fluids. ${ }^{6}$

\footnotetext{
a) Author to whom correspondence should be addressed. Electronic mail: gh.eslami@tabrizu.ac.ir. Telephone: (+98) 3016044.

b) Email: esmzadeh@ abrizu.ac.ir

c) Email: alberto@us.es
} 
Another interesting potential application of similar configurations is the heat transfer enhancement in fluid flow by the use of micron sized solid particles dispersed in the liquid bulk. Bologa et al..$^{7}$ studied the heat transfer enhancement caused by the electric field induced motion of solid particles in a gas-solid suspension. Added solid particles can improve the heat transfer rate between a fluid flow and a solid surface by advection, and disturbing and thinning the laminar sub-layer in the wall vicinity. ${ }^{8}$ Dispersed particles would improve turbulence intensity of a flow up to $100 \%$ when their size is of the order of the turbulence length scale. ${ }^{9}$ A theoretical study of local heat transfer enhancement through bombardment of a surface by spherical particles can be found in a paper by Murray. ${ }^{10}$ The electric force may help to overcome some difficulties faced by these technologies, like particle sedimentation and particle clogging. Augmentation of heat transfer rate in a dielectric fluid medium with the aim of electric force induced motion of added particles may become more important everywhere the gravitational force is weak, specifically in space transport systems. Also, heat transfer in colloidal suspensions with charged conductive particles is a field of interest. ${ }^{11}$

There are many papers on the subject of conductive particle motion induced by electric forces. Dascalescu and Mihailescu ${ }^{1}$ proposed a mathematical model for motion of a conductive particle exposed to DC corona field of coaxial wire-cylinder electrode system. Asano et al. ${ }^{4}$ conducted an analytical and experimental study on the acceleration and deceleration of a conductive particle in the DC electric field in a viscous fluid medium. They found a significant disagreement between their experimental data and the theoretical model prediction. They attributed this discrepancy to two factors. One is the hydrodynamic interaction between particle and electrode. The other is the fact that the particle obtains a charge smaller than expected after the particle-electrode contact. ${ }^{3,12}$ Recently, Jiang et al..$^{13}$ introduced an improved model for predicting the trajectories of conductive particles in roll-type electrostatic separator. Dascalescu et al..$^{14}$ conducted a numerical simulation of conductive particle behavior at the surface of a plate electrode affected by a DC corona field and employed the model to study the behavior of coal particles in fly-ash corona separators. Wang et al. ${ }^{15}$ fulfilled an experimental study about effects of conductive particles on the breakdown voltage of mineral oils and esters. Karunanayake and Hoshino ${ }^{16}$ accomplished a numerical simulation for predicting the electrostatic force acting on a conducting particle in the configurations of two parallel electrodes for the case in which the particle size is of the same order of the gap between electrodes. Besides the solid particle case, there is a large amount of studies carried out on the behavior of water droplet. Valuable experimental data are available from investigations of Jalaal et al. ${ }^{17}$ and Khorshidi et al. ${ }^{18}$

As is the case in the work of Asano, ${ }^{4}$ two main simplifications are usually done by previous researchers. The first one concerns the electric force being exerted on the particle. It is often considered a constant electric force equal to $E_{0} Q_{0}$, where $E_{0}$ is the uniform electric field far from the particle and $Q_{0}$ is the charge acquired by the particle after its collision with the electrodes. The second simplification is to neglect the hydrodynamic interaction between the particle and the electrode surface and simply employ the well-known Stokes' formula for the viscous drag $\left(6 \pi \mu R u_{p}\right)$.

The main goal of this paper is to construct an accurate model for the quantitative prediction of the motion of a conductive particle in the gap between two parallel electrodes. We take into account all the pertinent effects, including the influence of electrode presence on both electrical and hydrodynamic forces, as well as the microdischarge that takes place between an approaching conductive particle and the counter electrode (the electrode with charges opposite to that of the particle). The electric force was calculated by an expression derived by Pérez ${ }^{19}$ in which the image forces (caused by image charges and dipoles) have been taken into account. Particle-wall hydrodynamic interaction was introduced to the problem using appropriate wall-effect functions to modify steady Stokes drag and the so called added mass forces acting on the particle. Also, the microdischarge effect on the particle motion has been included by accounting the changes of electric force (both in value and orientation) immediately after the phenomenon (before the particle-electrode collision).

\section{PROBLEM DESCRIPTION AND RELATED ISSUES}

Consider a conductive spherical particle with radius, $R(=D / 2)$, mass density, $\rho_{p}$, between two parallel electrodes (see Figure 1). We assume that the particle diameter is greater than $10 \mu \mathrm{m}$. This assumption allows us to neglect any adhesion force between the particles and the electrodes. ${ }^{20}$ The 


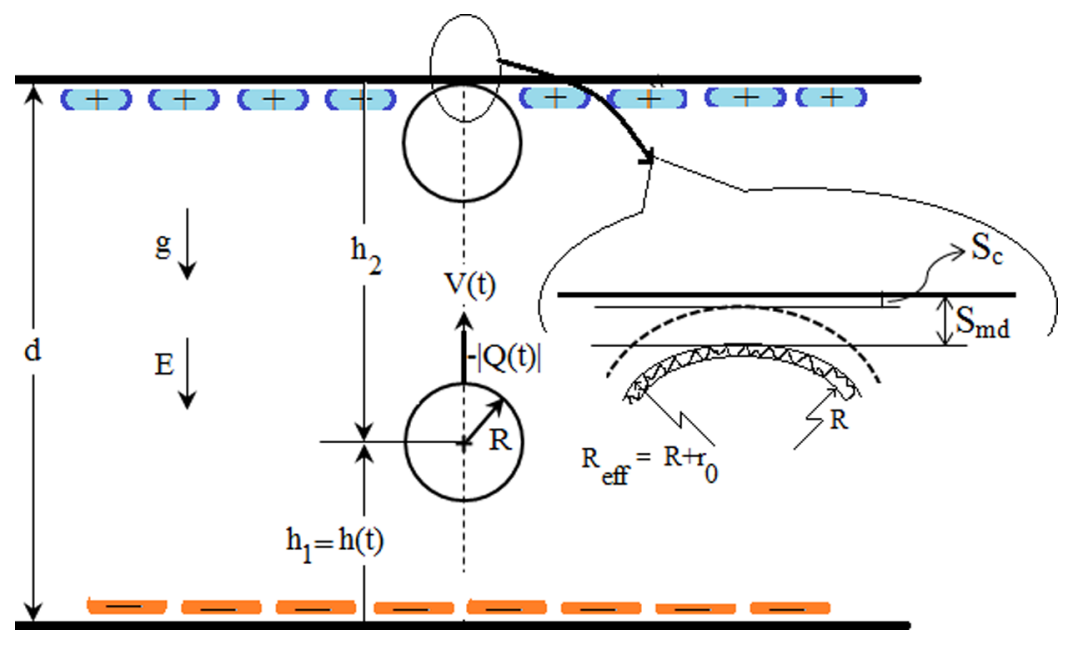

FIG. 1. Schematic presentation of the problem.

upper electrode has been connected to a high voltage supply with positive polarity while the lower one has been grounded. The electrodes are large enough to consider that the electric field is uniform everywhere except in a small region in the vicinity of the particle. This electric field has an average magnitude $E_{0}=\varphi / d$, where $d$ denotes the distance between the two electrodes and $\varphi$ is the applied electric potential. In what follows, it is described how the particle motion is started and how it is continued by the microdischarge mechanism.

\section{A. Particle starts to move}

When the particle is in mechanical contact with the lower electrode, it acquires an electric charge $Q_{0}$. Assuming that $R / d \ll 1$, the magnitude of $Q_{0}$, is given by the well-known formula ${ }^{21,22}$

$$
Q_{0}=\left(\frac{\pi^{2}}{6}\right) \cdot 4 \pi \varepsilon R^{2} E_{0}
$$

where $\varepsilon$ is the permittivity of the surrounding liquid. After charging, the particle is subjected to a total electric force,

$$
F_{e 0}=0.832 Q_{0} E_{0},
$$

which tends to lift it. When the sum of the buoyancy force $F_{b}$ and the electric one $F_{e 0}$ overcomes the gravitational force $F_{g}$, the particle detaches from the electrode and accelerates towards the upper electrode. After detachment, the particle behavior changes depending on the medium properties and charge exchange between the medium and the particle. Electrical charges of the particle would leak into the surrounding liquid due to the liquid electrical conductivity according to the formula ${ }^{23}$

$$
Q_{p}(t)=Q_{0} e^{-t / \tau_{c}},
$$

where $t$ is the time elapsed after the last detachment and $\tau_{c}=\varepsilon / \sigma$ is the charge relaxation time of the liquid ( $\sigma$ refers to the electrical conductivity of the liquid). From Eq. (3) it can be deduced that if the particle flight time, $t_{f i g h t}$, (the time interval between two successive particle rebound) becomes considerably lower than the charge relaxation time of the surrounding fluid, it is reasonable to ignore the charge leakage. Therefore, $\theta_{c}=t_{\text {flight }} / \tau_{c}$ is a dimensionless parameter that can affect the particle dynamics. Other dimensionless groups are presented in Section V C.

\section{B. Continuation of the motion through the microdischarge mechanism}

After detachment, while the conductive particle approaches to the upper electrode, the electric field between the particle and the electrode increases unboundedly until the surrounding dielectric liquid breaks down and a microdischarge takes place. ${ }^{20}$ During the microdischarge, first, the particle 
is neutralized and then it acquires the charge needed to be equipotential with the facing electrode. From the theoretical point of view, in the configuration under study, the charge transferred to the particle during the microdischarge event depends on its position at the discharging instant, $\bar{S}_{m d}$, and is obtained by

$$
Q_{t h}=4 \pi \varepsilon R^{2} E_{0}^{2}\left(\sinh ^{2} \alpha_{m d} \sum_{n=1}^{\infty} \frac{\cosh \left(n \alpha_{m d}\right)}{\sinh ^{2}\left(n \alpha_{m d}\right)}\right),
$$

where $\alpha_{m d}=\cosh ^{-1}\left(1+\bar{S}_{m d}\right)$ and $\bar{S}_{m d}=s_{\mathrm{md}} / R$ (see Eq. (37) in Ref. 19). The value of the function within parentheses in Eq. (4) equals to $\pi^{2} / 6$ in the limit $\bar{S}_{m d} \rightarrow 0$, applying $Q_{t h} \rightarrow Q_{0}$ (see Eq. (1)). However, this is an ideal theory, and in practice only a fraction of $Q_{t h}$ might be transferred to the particle (see Subsection VII A for more discussion).

Immediately after the microdischarge, the orientation and magnitude of the electric force $F_{e}$ change, and it acts as a breaking repulsion force on the approaching particle. Nevertheless, the particle may keep on its way of motion until it completely losses its inertia and changes the orientation of motion without physical contact with the electrode. Hereafter, we refer to this part of the particle motion as "post-microdischarge motion" which is discussed in Subsection VII B. However, if the initial inertia of the particle is great enough (usually big particles), the particle could collide with the electrode. In that case, a proper contact model is required to predict the behavior of the particle during and after the collision. In both the cases (with and without mechanical collision with the facing electrode), the particle rebounds and the motion is repeated in the same way.

There is a large amount of research dealing with microdischarge phenomena in different circumstances. ${ }^{24-26}$ It is rather difficult to exactly predict the distance between the particle and the electrode at which the breakdown happens ( $s_{m d}$ in Figure 1), because in addition to the electrical properties and purity of the interstitial fluid, it depends on the topographical details of the particle surface, as well. For example, even a tiny roughness on the particle and electrode surface would intensify the electric field significantly, since its size may be of the same order of magnitude as the gap between the particle and the electrode. However, as reported in Ref. 27, the microdischarge occurs at total electric field strengths (between the particle and the electrode) $E_{m d}$, similar to the breakdown electric field of the interstitial fluid.

\section{EFFECTIVE FORCES ACTING ON THE PARTICLE}

Ignoring adhesion forces between the particle and the electrodes, assuming that no charge injection takes place into the surrounding liquid from the surface of high voltage electrode and that no Electrohydrodynamic (EHD) flow sets up between the electrodes, the total external force acting on the particle will be

$$
\sum F_{\text {external }}=F_{e}+F_{f}+F_{g}+F_{b},
$$

where $F_{e}, F_{f}, F_{g}$, and $F_{b}$ denote the electric force, the fluid force, the gravitational force, and the bouncy force exerted on the particle, respectively.

In addition to the microdischarge phenomenon, the other main contribution of the present work pertains to the modification of $F_{e}$ and $F_{f}$ terms in Eq. (5). The hydrodynamic force $F_{f}$ consists of three distinguished terms, including steady drag, added mass, and history forces. Inclusion of the history force is extremely time consuming, and we ignore the last one in the present study. A more detailed discussion on the modification of the electric and hydrodynamic forces is presented in Secs. III A-III C.

\section{A. Electrostatic force}

In the parallel electrode system shown in Figure 1, the electrostatic force applied on a conductive sphere of radius $R$, having arbitrary charge $Q_{p}$, immersed in a medium of dielectric constant $\varepsilon$, can be calculated using three distinct methods:

- numerical solution of the Laplace equation 
- a Stokesian dynamic approach introduced recently by Drews et al. ${ }^{28}$

- the image charge method used by Pérez. ${ }^{19}$

The first method refers to the classical direct method and gives "exact" results, while the two other ones are approximate methods. The Stokesian dynamic approach is more accurate and also more complex than the image charge method. In this study we used the third method due to its simplicity, explicit expression, and less computational cost. It has been shown in Ref. 19 that for $R / d \leq 0.1$, its error is less than 3\%. According to this method the total electrostatic force consists of four distinct terms: charge-external field, charge-image charge, dipole-image dipole, and charge-dipole interaction. By adding these four terms, Pérez obtained an explicit expression for the total electric force as follows: ${ }^{19}$

$F_{e}(H)=\left\{\begin{array}{cc}4 \pi \varepsilon R^{2} E_{0}^{2} f_{0}(H-1)+Q_{p} E_{0} f_{1}(H-1)+\frac{Q_{p}{ }^{2}}{4 \pi \varepsilon R^{2}} f_{2}(H-1), & H \leq \frac{d}{2 R} \\ -4 \pi \varepsilon R^{2} E_{0}^{2} f_{0}\left(\frac{d}{R}-H-1\right)+Q_{p} E_{0} f_{1}\left(\frac{d}{R}-H-1\right)-\frac{Q_{p}{ }^{2}}{4 \pi \varepsilon R^{2}} f_{2}\left(\frac{d}{R}-H-1\right), & H>\frac{d}{2 R}\end{array}\right.$,

where $H=h / R$. $h$ is the distance between the particle center and the lower electrode. $E_{0}$ denotes the uniform electric field between the two parallel electrodes in the absence of the particle, and $f_{0}, f_{1}$ and $f_{2}$ are known functions of $H$ (see Ref. 19 for details). Other symbols are as before. The particle electric charge $Q_{p}=Q(t)$ can be obtained from Eq. (3) for any given time. Using Eq. (6), a modification factor can be introduced for the electric force as

$$
\lambda_{e}=\frac{F_{e}}{E_{0} Q_{0}}=\left\{\begin{array}{ll}
g_{1}\left(H, Q_{p}\right), & H \leq \frac{d}{2 R} \\
g_{2}\left(H, Q_{p}\right), & H>\frac{d}{2 R}
\end{array},\right.
$$

where $g_{1}\left(H, Q_{p}\right)$ and $g_{2}\left(H, Q_{p}\right)$ read

$g_{1}\left(H, Q_{p}\right)=\frac{6}{\pi^{2}} f_{0}(H-1)+\left(\frac{Q_{p}}{Q_{0}}\right) f_{1}(H-1)+\frac{\pi^{2}}{6}\left(\frac{Q_{p}}{Q_{0}}\right)^{2} f_{2}(H-1)$,

$g_{2}\left(H, Q_{p}\right)=-\frac{6}{\pi^{2}} f_{0}\left(\frac{d}{R}-H-1\right)+\left(\frac{Q_{p}}{Q_{0}}\right) f_{1}\left(\frac{d}{R}-H-1\right)-\frac{\pi^{2}}{6}\left(\frac{Q_{p}}{Q_{0}}\right)^{2} f_{2}\left(\frac{d}{R}-H-1\right)$.

Some numerical values of the functions $f_{0}, f_{1}$ and $f_{2}$ are given in Table I. The values in Table I illustrate the fact that these functions play an important role in the vicinity of the electrodes. This is caused by the electric field interaction between particle and the electrodes. This electric field interaction has been considered in the study of a conductive particle behavior in a roll-type corona-electrostatic separator by Dascalescue et al ${ }^{29}$ using an approximate formula,

$$
F_{e 0}=0.832 Q_{0} E_{0}\left[1+\frac{0.21(H-1)}{H-0.25}\right]
$$

TABLE I. Numerical values of $f_{0}, f_{1}$, and $f_{2}$ functions in vicinity of an electrode.

\begin{tabular}{lcrr}
\hline \hline$S=h / R-1$ & $f_{0}(S)$ & \multicolumn{1}{c}{$f_{1}(S)$} & \multicolumn{1}{c}{$f_{2}(S)$} \\
\hline 0.0001 & -220.0924 & 269.1032 & -81.7464 \\
0.001 & -34.1924 & 43.0022 & -12.9994 \\
0.01 & -5.4889 & 8.0143 & -2.3355 \\
0.1 & -0.7177 & 2.0687 & -0.4703 \\
1 & -0.03 & 1.0685 & -0.0671 \\
1.5 & -0.0100 & 1.0334 & -0.0414 \\
2 & -0.0047 & 1.0190 & -0.0283 \\
5 & $-2.9004 \times 10^{-4}$ & 1.0023 & -0.0070 \\
\hline \hline
\end{tabular}



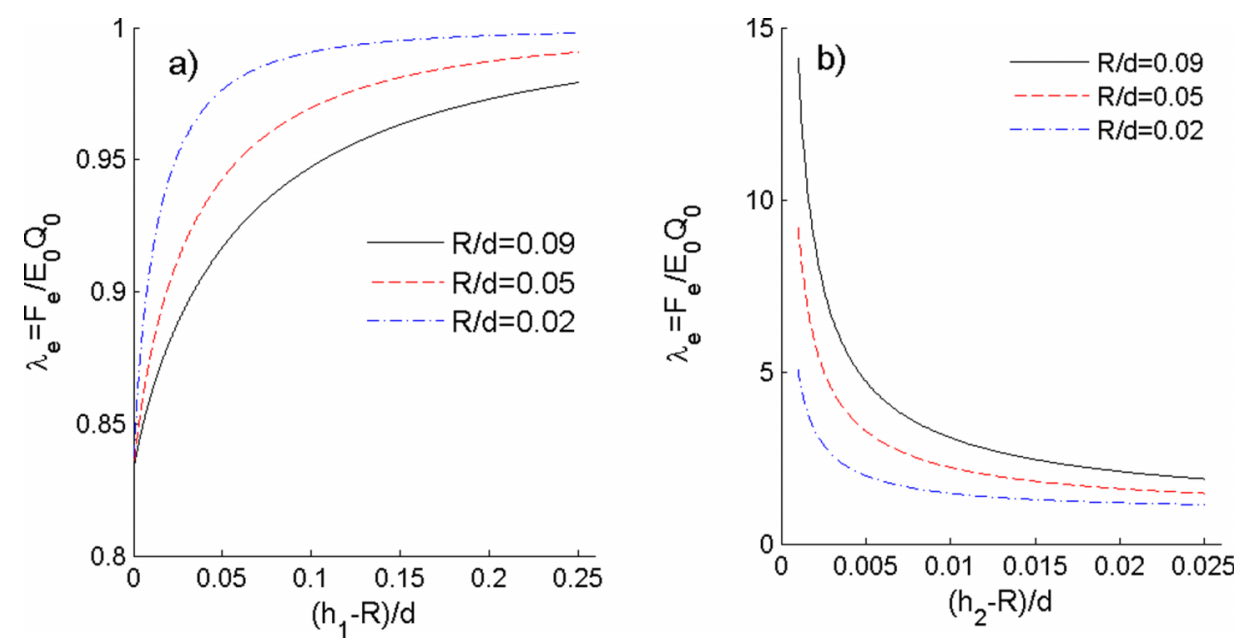

FIG. 2. Dimensionless electric force exerted on a detaching particle (a) and an approaching particle (b).

while the same formula was used in Ref. 20 for modeling of a conductive particle behavior in the parallel electrode system. In the present work, we use a more accurate one, Eq. (7a), which is derived specifically for the parallel electrode system and is accurate for $R / d \leq 0.1$.

It should be noted that the electric force applied on a detaching particle is completely different from the force on an approaching one. To illustrate this fact, we plot the force obtained from Eq. (7a) in Figure 2 for different ratios of the particle radius to the inter-electrode gap. As seen in Figure 2(a), all detaching particles see the same electric force predicted in Eq. (2) regardless of the ratio $R / d$. Also, as expected, deviation of the electric force from $E_{0} Q_{0}$ (Coulomb force) is more pronounced for bigger particles so that it reaches to nearly $25 \%$ of the gap between the two electrodes for $R / d=0.09$. Approaching particles, in contrast to the detaching ones, are attracted towards the electrode with an unbounded force, as shown in Figure 2(b).

\section{B. Hydrodynamic force exerted on the particle}

In order to determine the total force exerted on a moving particle by the surrounding fluid, it is needed to solve the continuity and Navier-Stokes equations with the appropriate boundary and initial conditions. However, when the Reynolds number (based on the particle velocity and diameter) is sufficiently low $(\operatorname{Re} \ll 1)$, the nonlinear inertial terms can be ignored. In this way the Navier-Stokes equation is reduced to the unsteady Stokes flow equation. Landau and Lifshitz ${ }^{30}$ solved this equation for a spherical particle starting to move at $t=0$, in an infinite domain of fluid, using boundary conditions of no-slip on the particle surface $(\vec{u}=0$ at $\mathrm{r}=\mathrm{R})$ and free flow velocity in the far field $(\vec{u}=\vec{U}$ at $r \rightarrow \infty)$. They determined the total force applied on the particle by the surrounding fluid as ${ }^{31}$

$F_{f}=\frac{9 \mu v_{p}}{2 R^{2}}(V-U)+\frac{1}{2} \rho_{f} v_{p} \frac{d V}{d t}-\frac{3}{2} \rho_{f} v_{p} \frac{d U}{d t}+\frac{9 \rho_{f} v_{p}}{2 R} \sqrt{\frac{v_{f}}{\pi}} \int_{0}^{t}\left(\frac{d V}{d \tau}-\frac{d U}{d \tau}\right) \frac{d \tau}{\sqrt{t-\tau}}$,

where $V$ and $U$ denote the particle and fluid free velocity, respectively, $v_{p}$ is the particle volume, $\tau$ is a dummy time variable, and $d / d t$ is the Lagrangian time derivative following the particle. For a stagnant medium $(U=0)$, Eq. (9) simplifies to

$$
F_{f}=\frac{9 \mu v_{p}}{2 R^{2}} V+\frac{1}{2} \rho_{f} v_{p} \frac{d V}{d t}+\frac{9 \rho_{f} v_{p}}{2 R} \sqrt{\frac{v_{f}}{\pi}} \int_{0}^{t} \frac{d V}{d \tau} \frac{d \tau}{\sqrt{t-\tau}} .
$$

The first term in Eq. (10) is the well-known Stokes drag,

$$
F_{s d}=\frac{9 \mu v_{p}}{2 R^{2}} V=\frac{24}{R e} \frac{1}{2} \rho_{f} A V^{2}=6 \pi \mu R V,
$$


and the second term is known as the conventional added (or virtual) mass force. This term is due to the particle acceleration in the fluid medium. Indeed, the added mass is an inertia added to the moving particle because it must accelerate (or decelerate) some volume of ambient fluid during its motion. And the last one is the history force (or fading memory force) that is often called the Basset force. It is caused by a temporary perturbation in the flow field created due to additional vortices at the solid particle surface which result from the particle acceleration. In other terms, the unsteady surface motion interacts with the developing boundary layer on the particle surface and results in a viscous force additional to the steady motion one. Further information about the concept and impact of the history force on the particle motion in various circumstances can be found in Refs. 31-37. Particle to fluid density ratio $\left(\rho_{p} / \rho_{f}\right)$ is a key parameter dictating the history force effect on the particle motion. The smaller $\rho_{p} / \rho_{f}$, the larger the history force effect. We did not account for the history force in this study.

\section{Particle-electrode hydrodynamic interaction (wall effect)}

The particle motion in the vicinity of a solid wall is influenced by the wall. As a particle approaches a solid wall, the interstitial liquid is squeezed out from the gap between the particle and the wall, increasing the value of the hydrodynamic force on the particle. ${ }^{38}$ The wall changes the form and pattern of streamlines around the moving particle and subsequently affects all terms of the hydrodynamic force acting on the particle. In this section, we present the latest available modification functions of wall effect on the steady drag and added mass forces acting on a moving spherical particle. Note that the wall effect on the particle motion depends on the orientation of its motion with respect to the wall, as well as on the Reynolds number. Here, only motion perpendicular to the wall (towards or away from) is considered.

To take into account the wall effect, a common practice is to introduce a correction factor $\lambda_{w}$, such that the steady Stokes drag force, $F_{s d}=6 \pi \mu R U$, is modified as

$$
F_{d}=\lambda_{w} F_{s d}
$$

\section{Wall effect on the steady drag for low Reynolds numbers $\left(\lambda_{s}\right)$}

For a spherical particle moving perpendicularly towards or away from a solid plane wall with constant velocity in a stagnant viscous medium having Reynolds number much less than unity, the wall correction function, $\lambda_{S}$, was derived independently by Maude ${ }^{39}$ and Brenner ${ }^{40}$ by solving the quasi-steady Stokes equation in a bi-spherical coordinate,

$$
\lambda_{S}=\frac{4}{3} \sinh (\alpha) \sum_{n=1}^{\infty} \frac{n(n+1)}{(2 n-1)(2 n+3)}\left[\frac{2 \sinh (2 n+1) \alpha+(2 n+1) \sinh 2 \alpha}{4 \sinh ^{2}\left(n+\frac{1}{2}\right) \alpha-(2 n+1)^{2} \sinh ^{2} \alpha}-1\right],
$$

where $\alpha=\cosh ^{-1}(H)$ and the subscript $S$ in $\lambda_{S}$ refers to the Stokes flow. It is important to note that although $\lambda_{S}$ has been derived disregarding the inertial terms in the Navier-Stokes equation, as Brenner ${ }^{40}$ discussed in his seminal work, its validity goes substantially above 0.5 , the value normally cited as the upper limit of Stokes' law for an unbounded fluid. In this study the instantaneous Reynolds number is considerably low near the electrodes but it grows up to $1 \sim 2$ in the middle of the interelectrode distance.

The following function gives a very good approximation to the exact formula for the entire range of gap between the particle surface and the wall $\left(S=h_{1} / R-1\right.$ for the lower electrode and $S=h_{2} / R-1$ for the upper one),

$$
\lambda_{S}=1+\frac{1}{S}+\frac{3}{19 S+4}
$$

The wall effect correction function obtained from Equations (13) and (14), as well as experimental data of Adamczyk et al., ${ }^{41}$ has been depicted in Figure 3(a). As it is seen, there is a very good agreement among three of them. Therefore, Eq. (14) can be used in calculations of the proposed model instead of the complicated formula Eq. (13) without losing accuracy. Figure 3(b) indicates 

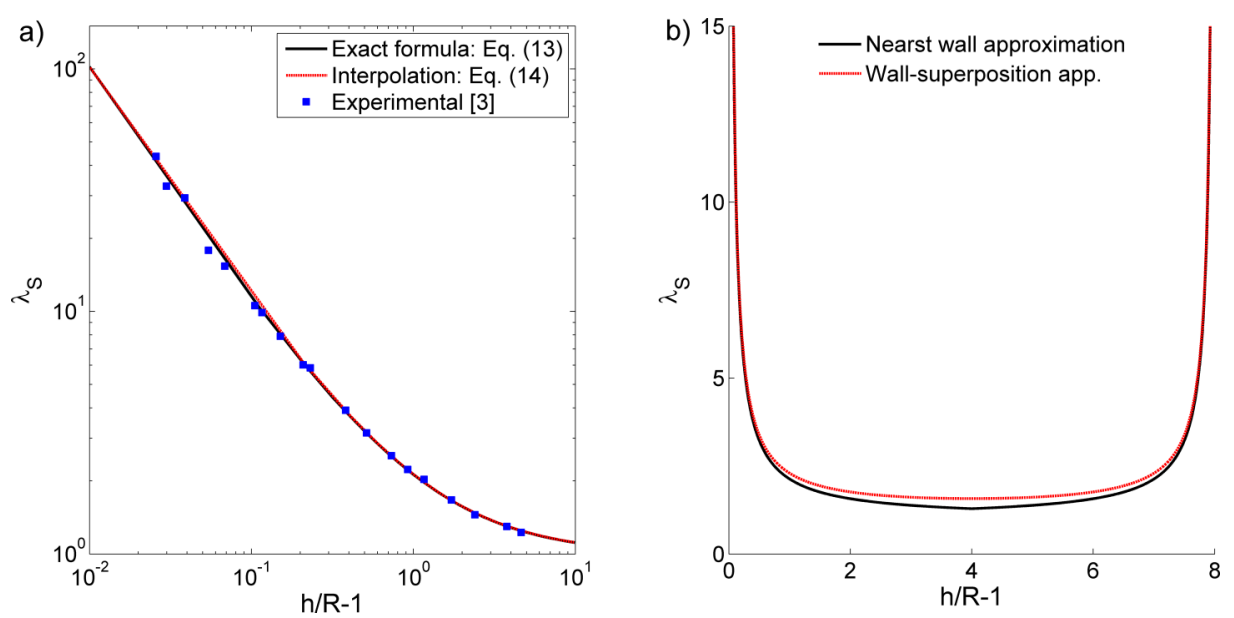

FIG. 3. Wall correction function. Comparison among results of Eq. (13), Eq. (14), and experimental data (a). Comparison between the nearest wall and wall-superposition approximations (b).

that the particle-wall interaction starts to influence the particle motion at a distance equal to nearly ten times the particle diameter. This means that the drag force acting on a typical particle with $D=1 \mathrm{~mm}$ submerged in a parallel electrode system with $10 \mathrm{~mm}$ inter-electrode distance will be continuously affected by the presence of electrodes via the wall effect on the whole gap. Hence, it seems that $\lambda_{S}$ plays an important role in the up and down motion of conductive particle submerged in a viscous liquid.

In order to take into account the simultaneous influence of both electrodes on the particle motion, one approximate approach is to superimpose the effect of both single electrodes,

$$
\lambda_{\text {sup }}=\lambda_{1}+\lambda_{2}-1,
$$

in which $\lambda_{1}$ and $\lambda_{2}$ are the wall effect correction functions of the nearest and farthest electrodes, respectively. The unity has been subtracted from the right side of Eq. (15) in order to avoid the double counting of the drag force applying on the particle in an unbounded medium. Since the governing equations for the creeping flow (steady Stokes equations) are linear, it seems that the wall-superposition approximation is a reasonable method to take into account the simultaneous influence of both electrodes. The nearest wall and wall-superposition approximations have been compared in Figure 3(b) for $R=1 \mathrm{~mm}$, and $d=10 \mathrm{~mm}$. As it is clear, they give the same magnitudes in the vicinity of the wall. The wall-superposition approximation predicts slightly greater values in the central region.

\section{Wall effect on the added mass force $\left(\lambda_{a m}\right)$}

As it has been said, the added mass force is due to the acceleration of the fluid caused by the particle motion. Obviously, when a particle is accelerated in an unbounded medium, the surrounding fluid can displace freely in every direction. However, the existence of a wall in the vicinity of the particle would restrict the displacement of the fluid, causing an increase of the added mass force. In spite of this consideration, it has been shown that, in the case of a spherical particle normally approaching to a solid plane wall, the added mass enhancement is small. For example, in an inviscid flow, the wall effect imposes a factor of $(1+0.375 / H)$ to the unbounded one, corresponding to a maximum enhancement of $37.5 \% .{ }^{33}$ Most recently, Simcik and Ruzicka ${ }^{42}$ conducted a 3D numerical simulation of full Navier-Stokes equations and obtained an accurate correlation for the added mass force acting on a spherical particle near a solid wall as

$$
F_{a m}=\left(\frac{1}{2}+\frac{0.1785}{H}\right) \rho_{f} v \frac{d V}{d t},
$$


which gives a wall modification factor as

$$
\lambda_{\text {am }}=1+\frac{0.357}{H}
$$

We use Eq. (17) as the wall modification factor of the added mass force in our calculations everywhere is needed.

It is worth to note that according to Eq. (17) the maximum value of $\lambda_{a m}$ is equal to 1.357 , which corresponds to the moment of contact between the particle and the wall (i.e., $H=1$ ). Therefore, based on the analysis of Simcik and Ruzicka, ${ }^{42}$ the added mass force will increased just up to $37 \%$ which is not much in comparison with the wall effect on the steady drag (see Eq. (13) or Eq. (14)). So it seems that the steady drag force modified by the wall correction function $\left(\lambda_{S}\right)$ is completely dominant near the wall and wall effects on the added mass forces can be reasonably neglected. However, we did include it in our calculations in order to give a quantitative comparison.

\section{PARTICLE-WALL COLLISION AND REBOUNDING PROCESSES}

Particle-wall as well as particle-particle collisions have an effective impact on liquid-solid flows because they influence inter-phase transportation phenomena. ${ }^{43}$ Particularly, particle-wall collisions would affect the heat transfer rate between a submerged surface and its surrounding medium by thinning the thermal and viscous boundary sublayers. The change in particle velocity after rebounding defines the restitution coefficient as

$$
k_{r}=\frac{V_{r}}{V_{i}},
$$

where $V_{i}$ and $V_{r}$ denote the particle velocity before and after a rebound, respectively. It should be noted that the impact velocity in a viscous medium is defined in a somewhat different way from that of a dry collision. In the viscous case, contrary to the dry collision case, the impact velocity is not the particle velocity in the moment just before the real physical contact. What is taken as the impact velocity is the maximum particle velocity before the rebound. In a gaseous environment, the maximum particle velocity is the same as the particle velocity at the real contact. This is not true for a viscous collision due to a sudden and sharp decrease in the particle velocity right prior to its collision with the wall. ${ }^{44,45}$ Such an enormous reduction in the approaching particle velocity is originated from the particle-wall interaction, which is brought about by a lubrication effect due to the interstitial liquid and can be quantified by the correction function, $\lambda_{S}$ (for the Stokes flow), represented in Eq. (13). The restitution coefficient is often expressed as a function of the ratio of the particle inertia to viscous forces, namely, the Stokes number,

$$
S t=\frac{1}{9} \frac{\rho_{p}}{\rho_{f}} \operatorname{Re}_{i}
$$

where $R e_{i}=\rho_{f} V_{i} D / \mu_{f}$ is the particle Reynolds number based on the impact velocity $V_{i}$. Almost all the experimental studies indicate that there is a critical Stokes number, $S t_{c} \approx 10$, below which no rebound occurs. ${ }^{44-47}$ In the current study, the Stokes number is quite smaller than $S t_{c} \approx 10$. Therefore, the restitution coefficient is not considered.

\section{MODELING APPROACH AND EQUATION OF MOTION}

In this section, first, we describe a model through which the microdischarge characteristics are treated. Then the particle equation of motion is presented. Also, we talk about how the model can be extended to intermediate Reynolds numbers. Finally, dimensionless form of the governing equations is obtained, and all the relevant dimensionless groups are introduced and discussed.

\section{A. Modeling of the electrostatic force}

In order to take into account the microdischarge, an additional parameter, denoted by $s_{m d}$, is introduced (see Figure 1). This parameter is the distance between the surface of the particle and the 


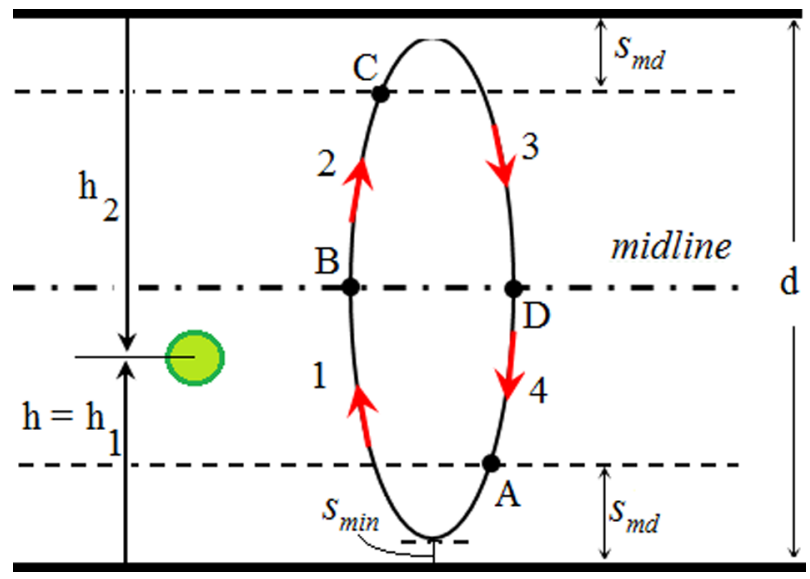

FIG. 4. The four segments considered in the computation of the particle trajectory.

electrode at which the electron avalanche caused by the local electric breakdown is produced. For the computation of the electric force, it is convenient to divide the trajectory in four segments: $\mathrm{AB}$, $\mathrm{BC}, \mathrm{CD}$, and DA, as depicted in Figure 4. A and B are discharging points near the lower and upper electrode, respectively. The dimensionless electric force is calculated in each segment according to the structure below:

- point A to point B: use Eq. (7b) with $h=h_{1}$,

- from point B to point C: use Eq. (7c) with $h=h_{2}$,

- from point C to point D: use Eq. (7b) with $h=h_{2}$,

- from point D to point A: use Eq. (7c) with $h=h_{1}$.

Based on the structure above and Eqs. (7b) and (7c), the dimensionless electric force reads

$$
\begin{aligned}
& \text { for } V \geq 0: \lambda_{e}\left(h, Q_{p}, s_{m d}\right)=\left\{\begin{array}{ll}
g_{1}\left(h, Q_{p}\right) & \text { for } h \leq d / 2 \\
g_{2}\left(d-h, Q_{p}\right) & \text { for } d / 2<h<d-\left(s_{m d}+R\right) \\
-g_{1}\left(d-h, Q_{p}\right) & \text { for } h \geq d-\left(s_{m d}+R\right)
\end{array}\right\}, \\
& \text { for } V<0: \lambda_{e}\left(h, Q_{p}, s_{m d}\right)=\left\{\begin{array}{ll}
-g_{1}\left(d-h, Q_{p}\right) & \text { for } h \geq d / 2 \\
-g_{2}\left(h, Q_{p}\right) & \text { for } s_{m d}+R<h<d / 2 \\
g_{1}\left(h, Q_{p}\right) & \text { for } h \leq s_{m d}+R
\end{array}\right\}
\end{aligned}
$$

where $\lambda_{e}$ is a positive value when the particle travels from the lower discharging point to the upper one (in the path $\mathrm{A}$ to $\mathrm{C}$ in Figure 4) and a negative value when it moves from the upper discharging point to the lower one (in the path $\mathrm{C}$ to $\mathrm{A}$ in Figure 4). Thus it can be deduced that during the upward motion when the particle arrives to the point $\mathrm{C}$, the microdischarge takes place and the electric force direction changes suddenly. As a result, the negative acceleration applying on the particle tends to prevent the particle from more approaching to the near electrode. This situation happens again in the downward motion when the particle reaches to the lower discharging point, $\mathrm{A}$. A typical microdischarge phenomenon near the lower and upper electrodes can be seen in Figure 5.

\section{B. The particle equation of motion}

The motion of a particle in a viscous fluid, which subjected to an electric field, is governed by

$$
m_{p} h^{\prime \prime}(t)=\vec{F}_{f}+\vec{F}_{e}+\vec{F}_{g}+\vec{F}_{b},
$$

where $h^{\prime \prime}(t)=d^{2} h(t) / d t^{2}$. With the above considerations, this equation can be rewritten as

$$
\left(m_{p}+\lambda_{a m} \frac{1}{2} \rho_{f} v_{p}\right) h^{\prime \prime}(t)=-\lambda_{w} \phi_{R e}(6 \pi \mu R) h^{\prime}(t)+\lambda_{e} E_{0} Q_{m d}-\left(m_{p}-\rho_{f} v_{p}\right) g .
$$



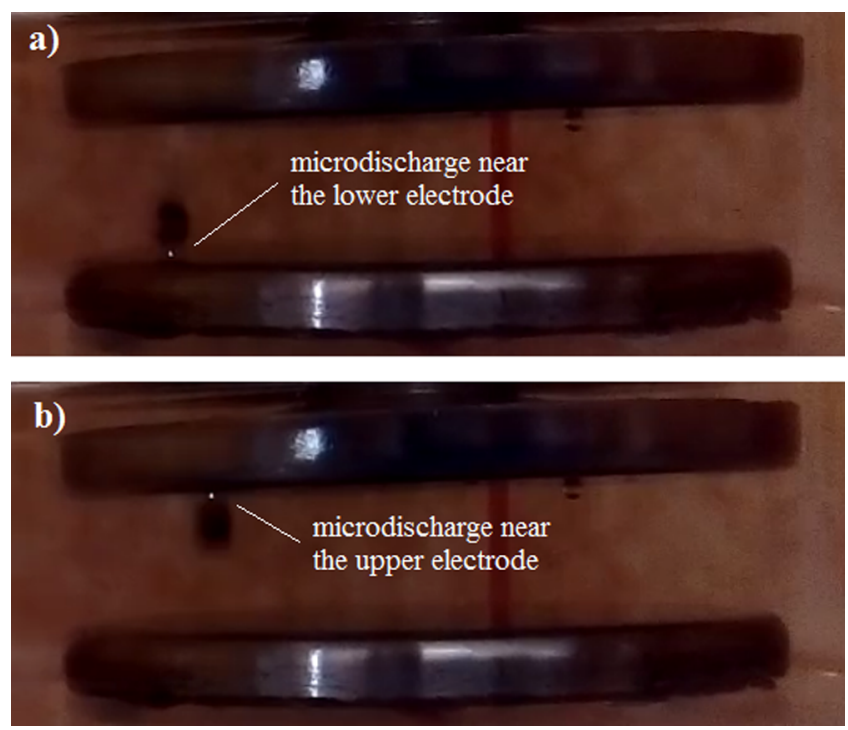

FIG. 5. A photograph showing the microdischarge phenomenon created by a $4 \mathrm{~mm}$ steel particle approaching to the lower electrode (a) and the upper electrode (b). The particle has been submerged in silicon oil and subjected to $\mathrm{E}=1 \mathrm{MV} / \mathrm{mm}$.

The parameter, $Q_{m d}$, is the amount of electrical charge acquired by the particle during the particleelectrode "contact" (we put the word contact between quotation marks to emphasize that it is not necessarily the mechanical contact). As we mentioned earlier in Section II B, in practice, $Q_{m d}$ might be only a fraction of the corresponding theoretical value, $Q_{t h}$ given in Eq. (4), rather than being exactly the same as it. However, to the best knowledge of the authors, there is no theory to determine that $Q_{m d}$ equals to what fraction of $Q_{t h}$. In Subsection VII C, we will describe an approach by which the exchanged charge, $Q_{m d}$, can be obtained from the experimental position-time data.

The function $\phi_{R e}=1+0.15 R e^{0.687}$ is commonly used to modify the Stokes' drag force to include intermediate Reynolds numbers $(0.1<\mathrm{Re}<1000) .{ }^{48}$ Also, an appropriate wall correction function is needed to account the wall effect on the hydrodynamic force in high or intermediate Reynolds number regime. One would use the following Cox-Brenner ${ }^{49}$ function:

$$
\lambda_{C-B}=\frac{1}{S}\left[1+\frac{1}{5}\left(1 \pm \frac{1}{2} \frac{R U}{v_{f}}\right) S \ln \frac{1}{S}\right],
$$

where positive and negative signs are for the approaching and rebounding particles, respectively. However, it should be noted that Eq. (23) is held just for $S \ll 1$. To the best knowledge of the authors, there is no explicit expression for the wall correction factor when $S$ is not very smaller than unity. One possible expensive way is numerical solution of the full Navier-Stokes equations, but another approximate approach for higher Reynolds number is matching $\phi_{R e}$ and $\lambda_{C-B}$ in a point $\left(S_{\text {switch }}\right)$, where the two correction factors $\phi_{R e}$ and $\lambda_{C-B}$ converge to the same magnitude. This method has been described in Ref. 50. In spite of agreement between predictions of this method and experimental data of Ref. 50, one should be careful when using it because, firstly, in this approach the wall correction factor has been restricted to very small values of $S$, and secondly, as we tried a smooth transition from $\phi_{R e}$ to $\lambda_{C-B}$ at $S_{\text {switch }}$ is not guaranteed always.

For low Reynolds number, $\lambda_{w}=\lambda_{S}$. Also, $\phi_{R e}$ can be removed from Eq. (22), accordingly we have

$$
\left(m_{p}+\lambda_{a m} \frac{1}{2} \rho_{f} v_{p}\right) h^{\prime \prime}(t)=-\lambda_{S}(6 \pi \mu R) h^{\prime}(t)+\lambda_{e} E_{0} Q_{m d}-\left(m_{p}-\rho_{f} v_{p}\right) g,
$$

where the modification factors $\lambda_{e}, \lambda_{S}$ and $\lambda_{a m}$ are given in Equations (7a), (13) or (14), and (17). Note that in order to remove the effect of any modification factor it suffices to make it equal to 1 in Eq. (24). The particle velocity is given by $V(t)=d(h(t)) / d t$ (note that $V>0$ and $V<0$ stand for the upward and downward motion, respectively). 


\section{Dimensionless form of the governing equation and discussion}

Dimensionless form of Eq. (24) can be obtained by using appropriate scales for length, velocity, and time. Since the particle radius $(R)$ is used for calculating of all the relevant forces, it can be a suitable scale for the length. Also, the terminal velocity of a charged particle (with charge $Q_{0}$ ) which moves in an unbounded medium under the action of a uniform electric field, $E_{0}$, and Stakes drag is a good choice for the velocity scale, i.e., $V_{\infty}=E_{0} Q_{0} / 6 \pi \mu R$. After substitution of $Q_{0}$ from Eq. (1), it reads $V_{\infty}=\pi^{2} \varepsilon R E_{0}^{2} / 9 \mu$. Finally the time can be dimensionlessed by $t_{\infty}=R / V_{\infty}$ (i.e., $\theta=t / t_{\infty}$ ). Using these scales, Eq. (24) is dimensionlessed as

$$
\Pi_{I} \frac{d^{2} H}{d \theta^{2}}=-\lambda_{S} \frac{d H}{d \theta}+\lambda_{e}-\Pi_{g}
$$

where

$$
\Pi_{I}=\frac{\pi^{2}}{81}\left(\rho_{p}+\lambda_{a m} \frac{1}{2} \rho_{f}\right) \frac{\varepsilon R^{2} E_{0}^{2}}{\mu^{2}}
$$

and

$$
\Pi_{g}=\frac{2}{\pi^{2}} \frac{\left(\rho_{p}-\rho_{f}\right) g R}{\varepsilon E_{0}^{2}} .
$$

Surprisingly, $\Pi_{g}$ is exactly the same as the particle apparent weight to the electric force ratio, i.e., $\Pi_{g}=\left(m_{p}-m_{f}\right) g / E_{0} Q_{0}$. For conditions in which the up and down motion is possible, the dimensionless number $\Pi_{g}$ should lie in the range $0 \leq \Pi_{g}<1$. As $\Pi_{g} \rightarrow 1$, the particle apparent weight will more pronounced on the particle dynamics. Furthermore, it is clear in Eq. (27) that $\Pi_{g} \propto E_{0}^{-2}$. This means that for fixed conditions, as the electric field is strengthened, the particle behaves more similar to an equivalent buoyant particle. Also, since $\Pi_{I} \propto E_{0}^{2}$, the stronger electric field amplifies the impact of the particle inertia. Therefore, one should be careful in removing the particle inertia force from the equation of motion.

In addition to $\Pi_{I}$ and $\Pi_{g}$ there are two other dimensionless groups that originate from the electric force. One of them pertains to the position of the microdischarge event and the other to the magnitude of charge obtained by the particle through the microdischarge. These dimensionless groups are, respectively,

$$
\bar{S}_{m d}=\frac{s_{m d}}{R}
$$

and

$$
\Pi_{Q}=\frac{Q_{p}}{Q_{0}} .
$$

These parameters appear in the dimensionless electric force as $\lambda_{e}=\lambda_{e}\left(H, \Pi_{Q}, \bar{S}_{m d}\right)$ (see Eq. (20a)). $\bar{S}_{m d}$ pertains directly to the breakdown strength of the intestinal fluid. Unfortunately, details of the microdischarge phenomenon in the under study problem are not known yet, and up to now there is no theory to determine $\Pi_{Q}$ in a general case. More recently Drews et al. ${ }^{51}$ have discussed on this issue. Usually, $\Pi_{Q}$ is considered as a free parameter in the proposed models to adjust their results with experimental data. Generally, $\bar{S}_{m d}$ and $\Pi_{Q}$ may be different for different electrodes due to the difference in the topography and roughness height of the electrode surface. These groups together with $\theta_{c}=t_{\text {fight }} / \tau_{c}$ form at least five and at most seven dimensionless parameters governing a typical conductive particle dynamic in the under study configuration. Therefore

$$
H=\operatorname{function}\left(\theta, \theta_{c}, \Pi_{I}, \Pi_{g}, \bar{S}_{m d}, \Pi_{Q}\right) .
$$

\section{EXPERIMENTAL APPARATUS AND METHOD}

In addition to the experimental data available in the literature, we carried out some experiments for evaluation of the proposed model. A schematic diagram of the experimental setup is depicted in Figure 6. A two-parallel-electrode system was constructed inside a cubic plastic box with internal 


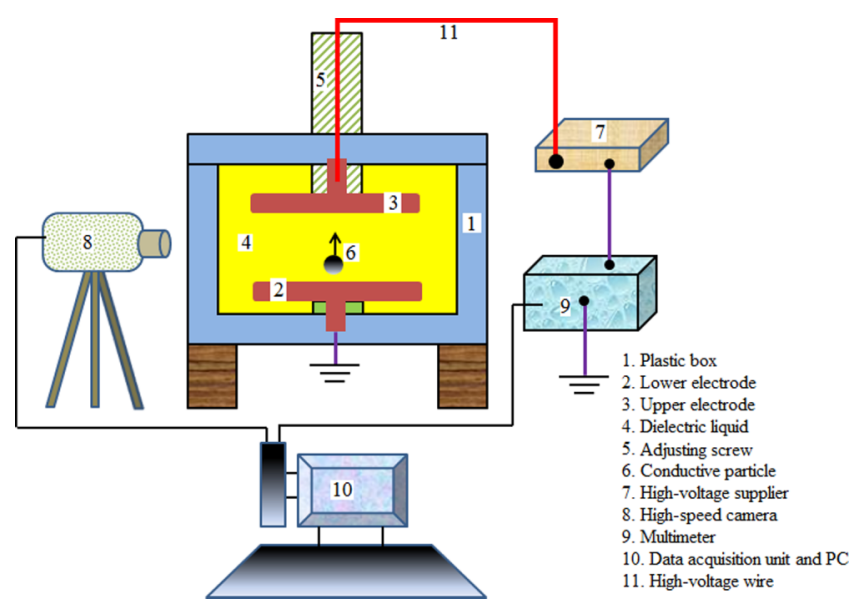

FIG. 6. Schematic view of the experimental setup.

dimensions of $150 \times 150 \times 80 \mathrm{~mm}$. The two flat stainless steel disks are of approximately $70 \mathrm{~mm}$ diameter and $5 \mathrm{~mm}$ thickness. One of the disks was fixed to the bottom of the plastic box, while the other one was hanged from the top of the box with a Teflon screw. The electrodes gap can be adjusted by the Teflon screw in the range of 0 to $50 \mathrm{~mm}$, with $0.1 \mathrm{~mm}$ accuracy. The edges of both electrodes were smoothed out carefully in order to prevent any charge injection into the surrounding liquid. A photograph of the test section is shown in Figure 7. The plastic box was filled with the working dielectric liquid, submerging the electrodes and the conductive particle. The upper electrode was connected to a DC high voltage power supply (SPELLMAN model: RHR4) with positive polarity and the lower one was properly grounded. The magnitude of the high voltage was controlled by an accurate high voltage probe having a resolution of $0.03 \%$ (VITREK, 4700 precision high voltage meter). A multimeter (Keithley 6517A) was used to measure continuously the electric current passing through the liquid. A camera recorded the multimeter panel along each test. Two spherical particles made of stainless steel with diameters of 2 and $0.5 \mathrm{~mm}$ and density of $7800 \mathrm{~kg} / \mathrm{m}^{3}$ and $7820 \mathrm{~kg} / \mathrm{m}^{3}$, respectively, were used throughout the tests. The particle motion was observed through the transparent side walls of the plastic box and recorded by a high speed video camera (PHOTORON, FASTCAM-ultima SE). The filming was carried out with 600 fps. MATLAB image processing toolbox was utilized whenever it was needed to process the frames.

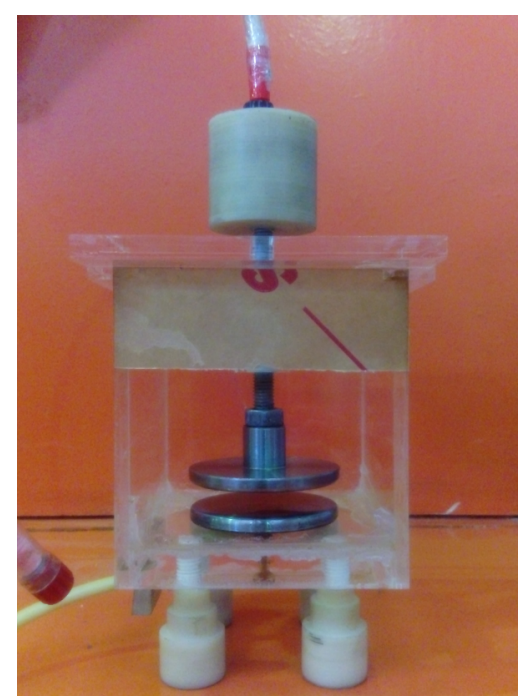

FIG. 7. Photograph of the test section. 
TABLE II. Parameters of the experimental cases.

\begin{tabular}{|c|c|c|c|c|}
\hline & Parameter & Case-1 (Ref. 3) & $\begin{array}{l}\text { Case-2 (Ref. } 3 \text { and } \\
\text { present work) }\end{array}$ & Case-3 (A, B and C) (present work) \\
\hline \multirow{6}{*}{ Dielectric liquid } & Oil name & Silicone oil (100 cSt) & Silicone oil (100 cSt) & $\begin{array}{l}\text { Silicone oil }(50 \mathrm{cSt})+\mathrm{A}: \text { No BA, B: } \\
0.25 \text { wt. \% BA, C: } 0.50 \text { wt. \% BA }\end{array}$ \\
\hline & $\rho_{f}\left(\mathrm{~kg} / \mathrm{m}^{3}\right)$ & 960 & 960 & 950 \\
\hline & $\mu(\mathrm{Pa} \mathrm{s})$ & 0.096 & 0.096 & 0.048 \\
\hline & $\varepsilon_{r}$ & 2.5 & 2.5 & 2.5 \\
\hline & $\sigma(\mathrm{S} / \mathrm{m})$ & $1.3 \times 10^{-12}$ & $1.3 \times 10^{-12}$ & $\begin{array}{c}\text { A: } 2.63 \times 10^{-12}, \mathrm{~B}: 1.30 \times 10^{-11}, \mathrm{C}: \\
2.62 \times 10^{-11}\end{array}$ \\
\hline & $\tau_{c}(\mathrm{~s})$ & 17.01 & 17.01 & A: $8.416, B: 1.702, C: 0.845$ \\
\hline \multirow{3}{*}{ Particle } & Material & Glassy carbon & Steel & Steel \\
\hline & $2 \mathrm{R}(\mathrm{mm})$ & 2 & 2 & 0.5 \\
\hline & $\rho_{p}\left(\mathrm{~kg} / \mathrm{m}^{3}\right)$ & 1500 & 7800 & 7820 \\
\hline \multirow{2}{*}{$\begin{array}{l}\text { Parallel electrode } \\
\text { system }\end{array}$} & $\varphi(\mathrm{kV})$ & 8 & 10 & 12 \\
\hline & $\mathrm{d}(\mathrm{mm})$ & 10 & 10 & 20 \\
\hline
\end{tabular}

\section{RESULTS AND DISCUSSION}

In this section, first, the magnitude of charge transferred to the particle by the microdischarge is discussed. Then, the effects of the microdischarge location $\left(s_{m d}\right)$ on the particle dynamics are presented based on the results obtained by solution of the particle equation of motion. Finally, a comparison is made between the model predictions and some experimental data of the literature as well as the present study. Input parameters and the dimensionless groups of all the studied cases are given, respectively, in Tables II and III.

\section{A. The charge transferred to the particle by the microdischarge}

As it was mentioned previously, in the configuration under study, the charge transferred to the particle during the microdischarge event, $Q_{m d}$, can be calculated by Eq. (4) according to the available theory. However, it seems that there is a contradiction in the literature about the amount of the exchanged charge. While Khayari et al..$^{23}$ and Tobazeon ${ }^{51}$ confirm the theoretical value, some other researchers have claimed that the exchanged charge is just some fraction of $Q_{t h}$. For instance, Drews et al. ${ }^{28-52}$ found the charge of $Q_{m d}=0.602 Q_{t h}$ and $Q_{m d}=0.66 Q_{t h}$ in agreement with their experimental data carried out on micron sized particles. In a similar way, Choi et al. ${ }^{12}$ reported that the experimental results of their study agree with their theoretical model if the acquired charge is considered as $40 \%-60 \%$ of its theoretical value (i.e., $\Pi_{Q}=0.4-0.6$ ). Surprisingly, in the current study, we showed that their results can be predicted by assuming $\Pi_{Q}=1$ for the upward motion and

TABLE III. Dimensionless parameters of all the studied cases characterizing the particle dynamic behavior.

\begin{tabular}{|c|c|c|c|c|c|}
\hline \multirow{2}{*}{$\begin{array}{l}\text { Experimental } \\
\text { cases }\end{array}$} & \multicolumn{5}{|c|}{ Dimensionless groups } \\
\hline & $\Pi_{I}$ & $\Pi_{g}$ & $\bar{S}_{m d}$ & $\Pi_{Q}$ & $\theta_{c}=\max \left\{t_{\text {flight }}\right\} / \tau_{c}$ \\
\hline Case 1 & 0.371 & 0.076 & 0.002 & $\begin{array}{c}1 \text { and } \\
0.81\end{array}$ & 0.010 \\
\hline Case 2 & 2.423 & 0.614 & 0.001 & $\begin{array}{c}1 \text { and } \\
0.85\end{array}$ & 0.015 \\
\hline Case $3 \mathrm{~A}$ & 0.219 & 0.429 & 0.001 & $\begin{array}{c}1 \text { and } \\
0.84\end{array}$ & 0.125 \\
\hline Case 3B & 0.219 & 0.429 & $\ldots$ & 1 & 1.968 \\
\hline Case $3 \mathrm{C}$ & 0.219 & 0.429 & $\ldots$ & 1 & 1.917 \\
\hline
\end{tabular}




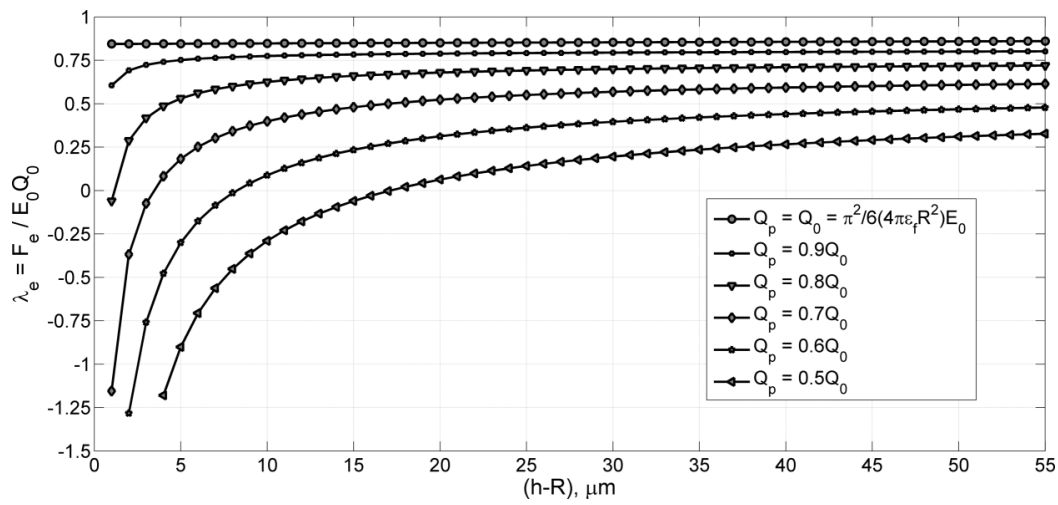

FIG. 8. The dimensionless electric force acting on a particle located very close to the electrode with the same-sign charge of value $Q_{p}$.

$\Pi_{Q}=0.81-0.85$ for the downward motion provided that the wall effect is taken into account (see Figures 16 and 17).

By careful review of the mentioned works, one can find out that the tested particle size and the acting forces are somewhat different. Here, we show that how the particle apparent weight would influence the amount of charge obtained by the particle-electrode contact. In order to clarify this point, we calculate the dimensionless electric force being exerted on the particle, $\lambda_{e}$, using Eqs. (7a) and (7b), assuming a smaller acquired charge in the range $0.6 Q_{0} \leq Q_{p} \leq Q_{0}$. The results are depicted in Figure 8 for a particle-electrode gap ranged from $1 \mu \mathrm{m}$ to $55 \mu \mathrm{m}$. When $Q_{p}$ is assumed to be less than $Q_{0}$, the electric force decreases considerably as the particle approaches to the electrode. Even, negative values are observed for $Q_{p} \leq 0.8 Q_{0}$, thus indicating an attractive force. For example, suppose an occurrence of the microdischarge at $s_{m d}=10 \mu \mathrm{m}$. If we assume that the particle acquires $Q_{p}=0.6 Q_{0}$, then the resulting force will be $F_{e} \approx 0.09 E_{0} Q_{0}$, which is much smaller than the force required for lifting off the particle. Also, assuming $Q_{p}=0.5 Q_{0}$ leads to an attraction force of value $F_{e} \approx-0.29 E_{0} Q_{0}$. Indeed, the lesser the acquired charge, the smaller the repulsive electric force. Obviously, this behavior is due to the image charges and dipoles. In fact, for a particle located at the vicinity of an electrode, as the particle charge decreases, the effect of image forces is more pronounced.

For a given particle-electrode gap, there is a definite value of $Q_{p}$ below which the particle will be attracted towards the electrode instead of being repelled. A three dimensional plot of $\lambda_{e}$ (see Figure 9) clarifies this issue. For example, if the charge transferred to the particle surface is less than $60 \%$ of its theoretical value (i.e., $\Pi_{Q} \leq 0.6$ ), it will be attracted to the near electrode whenever the dimensionless gap becomes smaller than $0.008(S \leq 0.008)$.

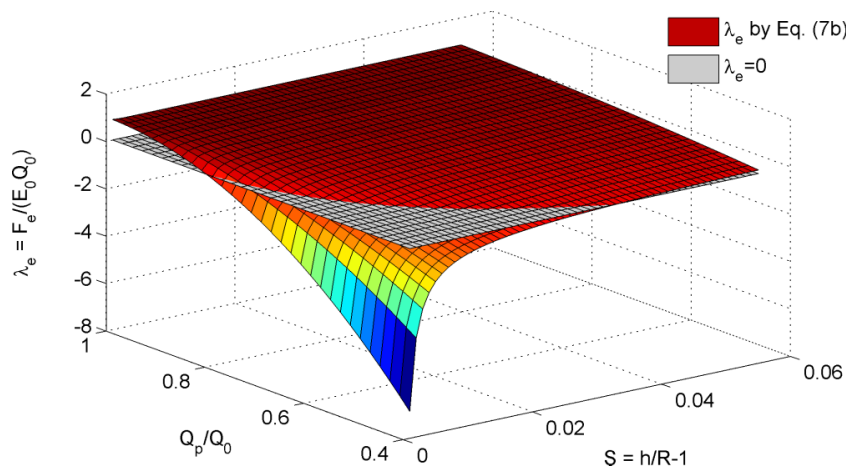

FIG. 9. Three dimensional plot of the dimensionless electric force as a function of the particle charge, $Q_{p}$, and the dimensionless gap, $S$. 
The minimum charge that the particle needs to be able to rebound (to lift) from the lower electrode $\left(\underline{Q}_{p \text {, min }}\right)$, can be determined by $F_{e} \geq F_{g}+F_{b}$, which gives $g_{1}\left(H_{\min }, Q_{p}\right) E_{0} Q_{0} \geq\left(m_{p}-\right.$ $\left.m_{f}\right) g$, where $H_{\min }$ is the particle center position at the instant of rebound (the instant that velocity of particle switch sign). This inequality can be converted to an equality by replacing $Q_{p}$ with $\underline{Q}_{p \text {,min }}$, i.e., $g_{1}\left(H_{\min }, \underline{Q}_{p \text {, min }}\right) E_{0} Q_{0}=\left(m_{p}-m_{f}\right) g$. Using Eqs. (27) and (28), it is simplified to $g_{1}\left(H_{\min }, \underline{Q}_{p, \min }\right)=\Pi_{g}$. After substituting $g_{1}\left(H_{\min }, \underline{Q}_{p, \min }\right)$ from Eq. (7b), it results in a second order polynomial equation as

$$
\frac{\pi^{2}}{6}\left(\frac{\underline{Q}_{p, \min }}{Q_{0}}\right)^{2} f_{2}\left(\bar{S}_{\mathrm{min}}\right)+\left(\frac{\underline{Q}_{p, \min }}{Q_{0}}\right) f_{1}\left(\bar{S}_{\text {min }}\right)+\frac{6}{\pi^{2}} f_{0}\left(\bar{S}_{\text {min }}\right)-\Pi_{g}=0,
$$

where $S_{\min }=H_{\min }-1$. A similar analysis is applicable for the particle bouncing (dropping) from the upper electrode. In this case the necessary condition is $F_{e} \geq-\left(F_{g}+F_{b}\right)$. Indeed, for possibility of the particle rebound from the upper electrode, the attractive electric force (negative force) should be necessarily weaker than the particle apparent weight. Such a constrain results in

$$
\frac{\pi^{2}}{6}\left(\frac{\bar{Q}_{p, \min }}{Q_{0}}\right)^{2} f_{2}\left(\bar{S}_{\min }\right)+\left(\frac{\bar{Q}_{p, \min }}{Q_{0}}\right) f_{1}\left(\bar{S}_{\min }\right)+\frac{6}{\pi^{2}} f_{0}\left(\bar{S}_{\text {min }}\right)+\Pi_{g}=0,
$$

where $\bar{Q}_{p \text {, min }}$ stands for the minimum charge required for the particle rebound from the upper electrode and $\bar{S}_{\min }$ denotes the dimensionless minimum gap between the particle and the electrode at the instant of rebound.

In Figure 10, the acceptable roots of Eqs. (31a) and (31b) are depicted as a function of $\bar{S}_{\min }$ for different values of $\Pi_{g}$. As it is observed, the minimum charge required for the particle while bouncing from the upper electrode might be considerably smaller than that of the lower electrode. Also, as expected, for a buoyant particle $\left(\Pi_{g}=0\right)$, there is no difference between $\underline{Q}_{p \text {, min }}$ and $\bar{Q}_{p \text {, min }}$. But, as $\Pi_{g}$ increases their difference grows significantly. For a given condition, a weighty particle may rebound from the upper electrode surface with less charge in comparison with a lighter one. However, it should be noted that such conclusion is correct only when the rebound takes place at the same $\bar{S}_{\min }$ for the two compared particles. Another remarkable result from Figure 10 relates to the effect of $\bar{S}_{\min }$ on the minimum required charge. As seen irrespective of both the electrode location (lower or upper one) and $\Pi_{g}$ value, lesser minimum separation at the instant of rebound requires more charge for the rebounding particle.

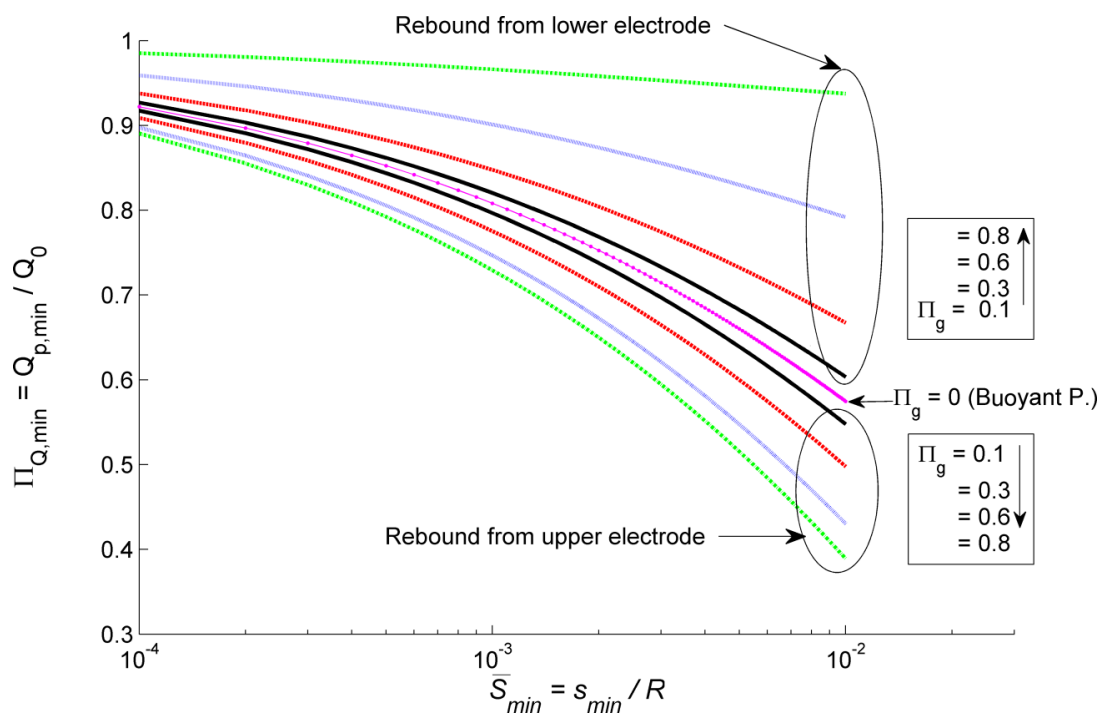

FIG. 10. The minimum required charge as a function of the minimum particle-electrode gap at the instant of rebound for different $\Pi_{g}$. 


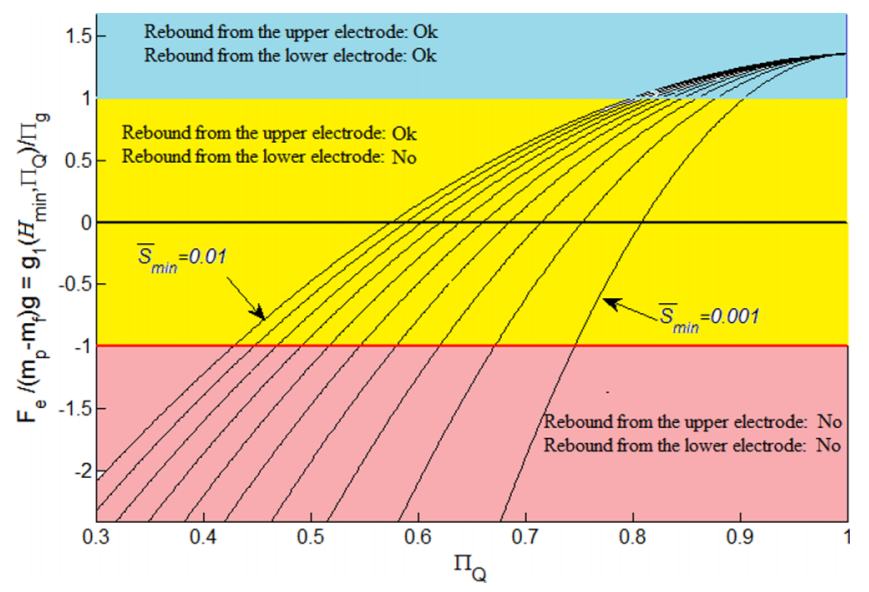

FIG. 11. Three distinct zones determining the possibility of the particle rebound from the upper and lower electrodes.

In Figure 11, the electric-to-apparent weight ratio is plotted versus $\Pi_{Q}=Q_{p} / Q_{0}$ for different values of the minimum particle-electrode gap. As a general rule, three distinct zones are detectable. In the zone $g_{1}\left(H_{\min }, Q_{p}\right) / \Pi_{g}>1$, the particle rebound from both the upper and lower electrode is possible. In the zone $g_{1}\left(H_{\min }, Q_{p}\right) / \Pi_{g} \leq-1$, the particle can rebound neither from the upper electrode nor from the lower one. In the middle zone $-1<g_{1}\left(H_{\min }, Q_{p}\right) / \Pi_{g} \leq 1$, the particle can separate from the upper electrode and drop low, but it cannot lift from the surface of the lower electrode.

\section{B. Post-microdischarge motion}

In order to reveal the effect of the microdischarge on the particle dynamics, first we studied the length of the post-microdischarge motion, $\Delta h_{p m d m}$, for input parameters of the case 1 in Table II. The particle trajectories were derived by solving Eq. (24) with the initial conditions: $h(0)=R+s_{\min }$ and $h^{\prime}(0)=0$ for different values of $s_{m d}$ and $E_{0}$ in the range $1 \mu \mathrm{m} \leq s_{m d} \leq 100 \mu \mathrm{m}$ and $0.6 \mathrm{MV} / \mathrm{m} \leq E_{0} \leq 1.5 \mathrm{MV} / \mathrm{m}$. Eq. (24) cannot be employed to predict the motion of a particle initially in full contact with the electrode, because as it is clear from Equation (13) or (14), the wall effect correction function is singular at $S=0$, representing no possible contact. In other words, since $S \rightarrow 0$ implies $\lambda_{S} \rightarrow \infty$, it is essential to use a minimum distance for the particle-wall gap, say $s_{\min }$. This strategy has been used by many researchers, for example, Drews et al. ${ }^{28}$ used $s_{\min }=0.0247 R(0.7 \mu \mathrm{m})$ in their modeling approach based on the assumption that the microdischarge takes place when the particle-electrode gap reduces to $0.7 \mu \mathrm{m}$ (i.e., $s_{m d}=0.7 \mu \mathrm{m}$ ). Indeed, they assumed that the particle motion is immediately reversed after the microdischarge. We evaluated this assumption and found out that it is correct when the microdischarge occurs in a point very close (in the order of one micron) to the next electrode (see Figure 13). However, as discussed in Subsection II B, an earlier microdischarge can take place for many reasons, particularly due to asperities on the particle and electrode surfaces. In that case, the "minimum distance" is not exactly the same as the "microdischarge point," and the particle can keep its direct motion towards the facing electrode. For such cases, the "minimum distance" should be determined by the post-microdischarge motion.

Variable time steps were utilized for solving the equation of motion. But in order to capture precisely the details of the particle trajectory and velocity, a constant time step size of $1 \mu$ s was used in the region close to the microdischarge position. Independency of the results was carefully checked against the time step size. Residual of a typical solution is depicted in Figure 12. It is in the order of $10^{-18}$ that indicates an excellent accuracy for the obtained numerical solution.

The results are shown in Figure 13 for the third microdischarge using logarithmic scale for $x$ and $y$ axes. As seen, both $s_{m d}$ and $E_{0}$ can influence the length of the post-microdischarge motion. A stronger electric field can drive the particle closer to the opposite electrode resulting in smaller $s_{\min }$. However, the wall hydrodynamic effect acts on the opposite side and prevents the particle from 


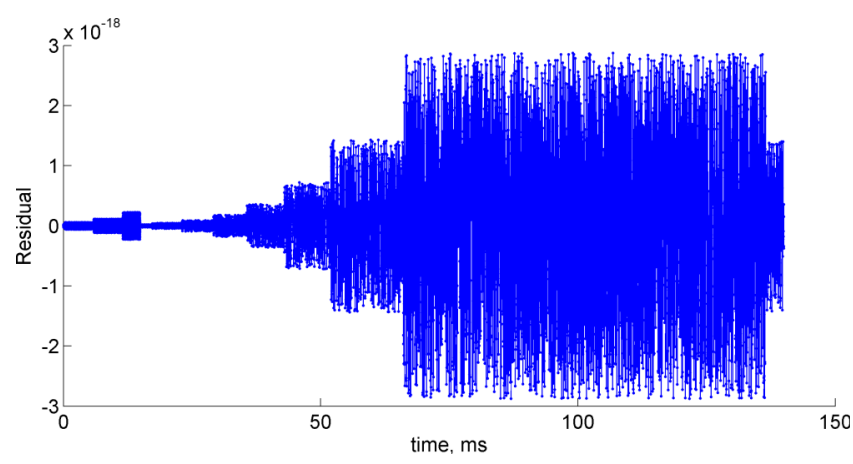

FIG. 12. Residual of a typical numerical solution in the present study.

more advancing. Indeed, the competition between $\lambda_{e}$ and $\lambda_{S}$ determines the position of the reverse point in the particle trajectory. It is clear from Figure 13 that $\Delta h_{p m d m}$ decreases significantly with decreasing $s_{m d}$, and $\Delta h_{p m d m} \approx 0$ for $s_{m d} \leq 1 \mu \mathrm{m}$ which conforms to the assumption of Drews et al. ${ }^{28}$ Generally, Figure 13 gives $s_{\min }=f\left(s_{m d}\right)$, where $f$ is a function between the microdischarge point position, $s_{m d}$, and the possible minimum particle-electrode separation, $s_{\min }$. This function can be used as the initial position of the particle in using the proposed model as

$$
\left.h\right|_{t=0}=R+s_{\min } \text { where, } s_{\min }=f\left(s_{m d}\right) .
$$

(Also, instead of $R+s_{\min }$, one would use an arbitrary initial position for the particle. But, in that case, it should be noted that the time evolution of the predicted trajectory is under the influence of the particle's initial position until the first microdischarge occurs. Thus, the experimental trajectory should not be compared with the first cycle of the predicted trajectory because the experimental data pertain to a consistent periodic bouncing motion of the particle.) We studied the trajectory of a glassy carbon particle for three different values of $s_{m d}: 100 \mu \mathrm{m}, 10 \mu \mathrm{m}$, and $1 \mu \mathrm{m}$. The results were derived by solving Eq. (24) with the initial conditions: $h(0)=R+s_{\min }$ and $h^{\prime}(0)=0$, where $s_{\min }$ was determined by $s_{\min }=f\left(s_{m d}\right)$ using Figure 13. All other parameters of the model were the same as in case 1 (see Table II).

The particle trajectory, velocity, and acceleration are depicted in Figures 14(a)-14(c), respectively. It is seen that the location of the microdischarge point can significantly influence the temporal evolution of the particle trajectory. Table IV shows some relevant quantitatives. Focusing on the case $s_{m d}=100 \mu \mathrm{m}$, as an instance, it is seen that right at $t_{m d}=101.970 \mathrm{~ms}$, the particle reaches the position which has been previously specified for the microdischarge phenomenon (point $\mathrm{C}$ in Figure 4). The particle velocity is almost $V_{m d}=47.14 \mathrm{~mm} / \mathrm{s}$, and it suddenly decreases to nearly zero in an interval of $\Delta t_{p m d m}=583 \mu \mathrm{s}$. During this interval, the particle experiences a huge negative

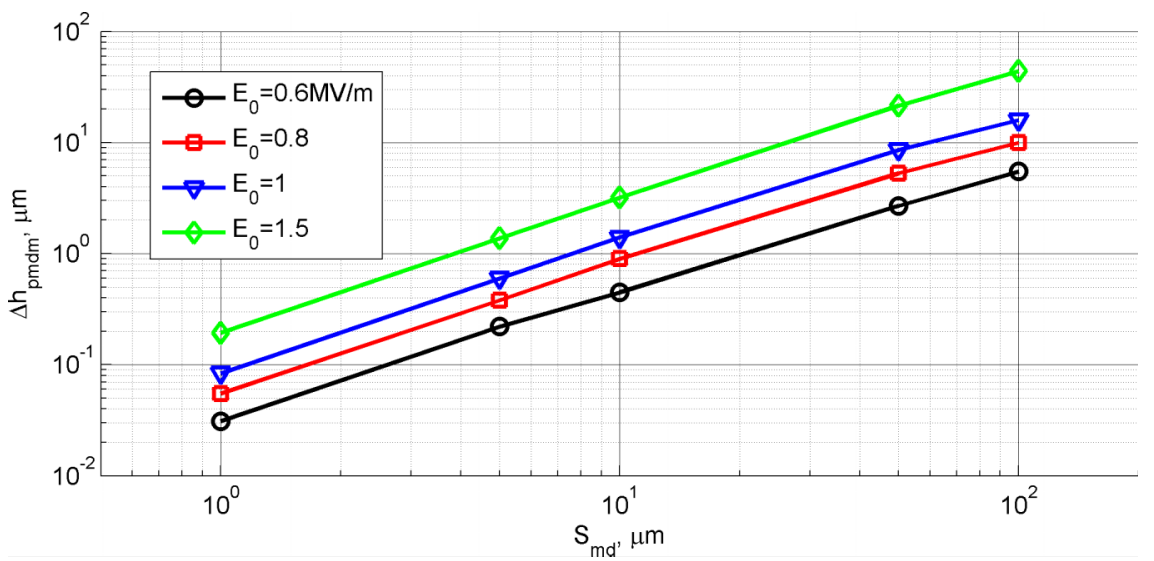

FIG. 13. The length of the post-microdischarge motion, $\Delta h_{p m d m}$, as a function of $s_{m d}$ and $E_{0}$. Other parameters are the same as case 1 in Table II. 

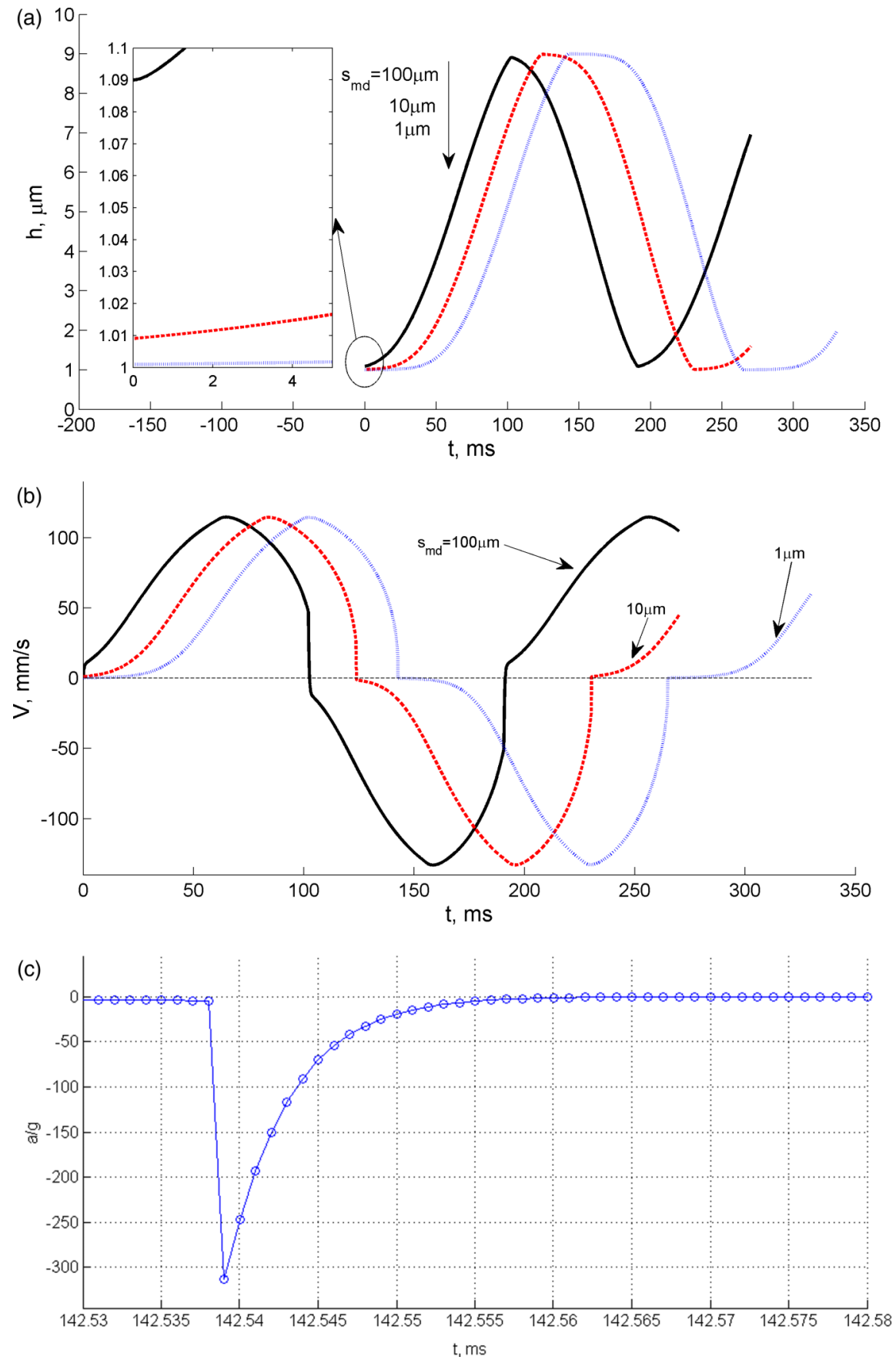

FIG. 14. Effect of the microdischarge phenomenon on the particle dynamics. (a) The particle trajectory; (b) temporal evolution of the particle velocity; (c) dimensionless acceleration of the particle for $s_{m d}=1 \mu \mathrm{m}\left(\bar{S}_{m d}=10^{-3}\right)$ immediately after the microdischarge near the upper electrode. Other input parameters are the same as in case 1.

acceleration and can move forward just $10 \mu \mathrm{m}\left(\Delta \mathrm{h}_{p m d m}=10 \mu \mathrm{m}\right)$. Consequently, its distance from the front electrode deceases from $100 \mu \mathrm{m}$ to almost $90 \mu \mathrm{m}$. Therefore, the proposed model predicts no physical contact between the particle and the electrode provided that the microdischarge happens at $s_{m d}=100 \mu \mathrm{m}$. This is in agreement with the experimental observation reported in Ref. 23. Overall behavior of the particle for the other two cases is similar to this case. But there is significant change in quantitative values of the pertaining parameters, particularly in $\lambda_{e}$ and $\Delta h_{p m d m}$. Comparison between values of these two parameters demonstrates that lesser $s_{m d}$ results in lesser $\Delta h_{p m d m}$. 
TABLE IV. Some parameters that characterize the particle dynamic behavior after the microdischarge near the upper electrode.

\begin{tabular}{lrrrccc}
\hline \hline$s_{m d}(\mu \mathrm{m})$ & $\bar{S}_{m d}$ & $\lambda_{e}$ & $t_{m d}(\mathrm{~ms})$ & $V_{m d}(\mathrm{~mm} / \mathrm{s})$ & $\Delta t_{p m d m}(\mu \mathrm{s})$ & $\Delta h_{p m d m}(\mu \mathrm{m})$ \\
\hline 100 & $10^{-1}$ & 3.24 & 101.970 & 47.14 & 583 & 10 \\
10 & $10^{-2}$ & 15.06 & 123.666 & 24.53 & 121 & 0.9 \\
1 & $10^{-3}$ & 83.87 & 142.516 & 13.78 & 16 & 0.05 \\
\hline \hline
\end{tabular}

This means that in a real case it is difficult to realize whether the particle rebound occurs after a real contact with the electrode or not.

As seen in Figure 14(b), in the discharging point (point $C$ in Figure 4), there is a sharp change in the particle velocity. This is also clear from Table IV. As an instance, for $s_{m d}=1 \mu \mathrm{m}$, the particle velocity decreases from $V_{m d}=13.78 \mathrm{~mm} / \mathrm{s}$ to $V \approx 0$ during an interval time of $\Delta t_{p m d m}=16 \mu \mathrm{s}$. Such a very rapid change in the velocity causes a large discontinuity in the particle acceleration as observed in Figure 14(c). This large discontinuity in the particle acceleration would cause the history force to be important in the rebounding trajectory. (Remember that the history force contains the acceleration term.)

An outcome of this section is the demonstration of the fact that the time and length scales of the particle post-microdischarge dynamic are, respectively, microsecond and micrometer. This is a very important result because such small time and length scales would influence the amount of charge obtained by the particle through the post-microdischarge motion. Indeed, since the microdischarge prevents the particle from a "mechanical" contact with the facing electrode, the charge exchange occurs throughout a thin layer of the interstitial liquid. In this case, the time scale of the charge exchange event might be comparable with that of the post-microdischarge motion. Based on such hypotheses, in addition to the particle inertia, even the chemical properties of the dielectric liquid plasma and roughness of the particle and electrode surfaces would play a role in the process of charge exchange during the microdischarge event. Further theoretical and experimental investigations are needed to shed more light on the details of this issue. An appropriate discussion can be found in Ref. 51. Due to very small time and length scales, a laser interferometry method ${ }^{55}$ is required for capturing the details of the post-microdischarge motion.

Also, it should be noted that after the microdischarge, the viscosity of interstitial liquid may change due to tiny bubbles which are produced because of vaporization of water content in the liquid. ${ }^{24}$ Also any possible sudden change in the particle temperature can influence the viscous drag force as explained in Ref. 53. We did not account for such effects in our study.

\section{Comparison with experimental data and discussion}

In order to use the proposed model, it is required to determine $Q_{m d}$. Here, first we explain a simple way to solve this issue. For applying this approach, at least three experimental data points, representing the particle position-time, are needed. These data points should be quite close to each other to save the accuracy. They would be extracted through image processing of the particle motion in the middle of the interelectrode distance. Assume that the three data points are $\left(h_{a}, 0\right),\left(h_{m}, t_{m}\right)$, and $\left(h_{b}, t_{b}\right)$, where, $h_{m} \leq d / 2$ and $0<t_{m}<t_{b}$. Using these data, we have $V_{a}=\left(h_{m}-h_{a}\right) / t_{m}, V_{m}=$ $\left(h_{b}-h_{m}\right) /\left(t_{b}-t_{m}\right)$, and $V_{m}^{\prime}=\left(V_{m}-V_{a}\right) / t_{m}$. By applying Eq. (24) for upward motion of the particle at $h=h_{m}$, after some simplification, it reads

$$
\left(m_{p}+\lambda_{a m, m} \frac{1}{2} \rho_{f} v_{p}\right) V_{m}^{\prime}=\lambda_{e, m} E_{0} Q_{0}-\left(m_{p}-\rho_{f} v_{p}\right) g-\lambda_{S, m}(6 \pi \mu R) V_{m} .
$$

The parameters $\lambda_{a m, m}, \lambda_{S, m}$ and $Q_{0}$ can be obtained, respectively, from Eqs. (17), (13), and (1) at $h=h_{m}$. By equating the dimensionless electric force $\lambda_{e, m}$, from Eqs. (33) and (7b), we have

$$
\frac{6}{\pi^{2}} f_{0}\left(H_{m}-1\right)+\left(\frac{Q_{m}}{Q_{0}}\right) f_{1}\left(H_{m}-1\right)+\frac{\pi^{2}}{6}\left(\frac{Q_{m}}{Q_{0}}\right)^{2} f_{2}\left(H_{m}-1\right)
$$




$$
=\frac{1}{E_{0} Q_{0}}\left[\left(m_{p}+\lambda_{a m, m} \frac{1}{2} \rho_{f} v_{p}\right) V_{m}^{\prime}+\left(m_{p}-\rho_{f} v_{p}\right) g+\lambda_{S, m}(6 \pi \mu R) V_{m}\right],
$$

where $Q_{m}$ stands for $Q$ at $h=h_{m}$. Now, the value of $\Pi_{Q}=Q_{m d} / Q_{0}$ can be easily obtained from Eq. (34). Since $Q_{m}=Q_{m d} \exp \left(-t_{m} / \tau_{c}\right)$, therefore

$$
Q_{m d}=\Pi_{Q} Q_{0} \exp \left(t_{m} / \tau_{c}\right) .
$$

The above mentioned procedure is used for obtaining $Q_{m d}$ from Eq. (35) whenever the proposed model is applied for the prediction of the particle trajectory.

Figure 15 shows a comparison between the predicted results of the model and the experimental data for the trajectory and velocity of a glassy carbon particle of $2 \mathrm{~mm}$ diameter and of $1500 \mathrm{~kg} / \mathrm{m}^{3}$ density submerged in silicon oil of $100 \mathrm{cSt}$ viscosity. The data labeled as "experimental" velocity in this and the following figures have not been measured directly. They have been calculated by first order forward step differentiation using the experimental data of the particle trajectory. All the required parameters are given in Table II under the column of case 1 . These values are those reported in Ref. 3. The dielectric constant and conductivity of the working liquid have not been given in the mentioned reference, so we assumed the sounded values of $\varepsilon=2.5, \sigma=1.3 E-12 \mathrm{~S} / \mathrm{m}$ which results in almost $\tau_{c}=17 \mathrm{~s}$ for the charge relaxation time. The three experimental data points $(4.26 \mathrm{~mm}, 88.18 \mathrm{~ms}),(5.00 \mathrm{~mm}, 96.2 \mathrm{~ms})$, and $(5.83,103.88 \mathrm{~ms})$ were selected from the particle trajectory graph given in Ref. 3 to be used for obtaining $Q_{m d}$ from Eq. (33). Thus, these data give $\left(h_{a}, 0\right)=(4.26 \mathrm{~mm}, 0 \mathrm{~ms}),\left(h_{m}, t_{m}\right)=(5.00 \mathrm{~mm}, 8.02 \mathrm{~ms})$ and $\left(h_{b}, t_{b}\right)=(5.83 \mathrm{~mm}, 15.81 \mathrm{~ms})$. Using these data, it is resulted that $Q_{m d}=0.99 Q_{0}$. This is in contradiction with $Q_{m d} \approx 0.4 Q_{0}-0.6 Q_{0}$
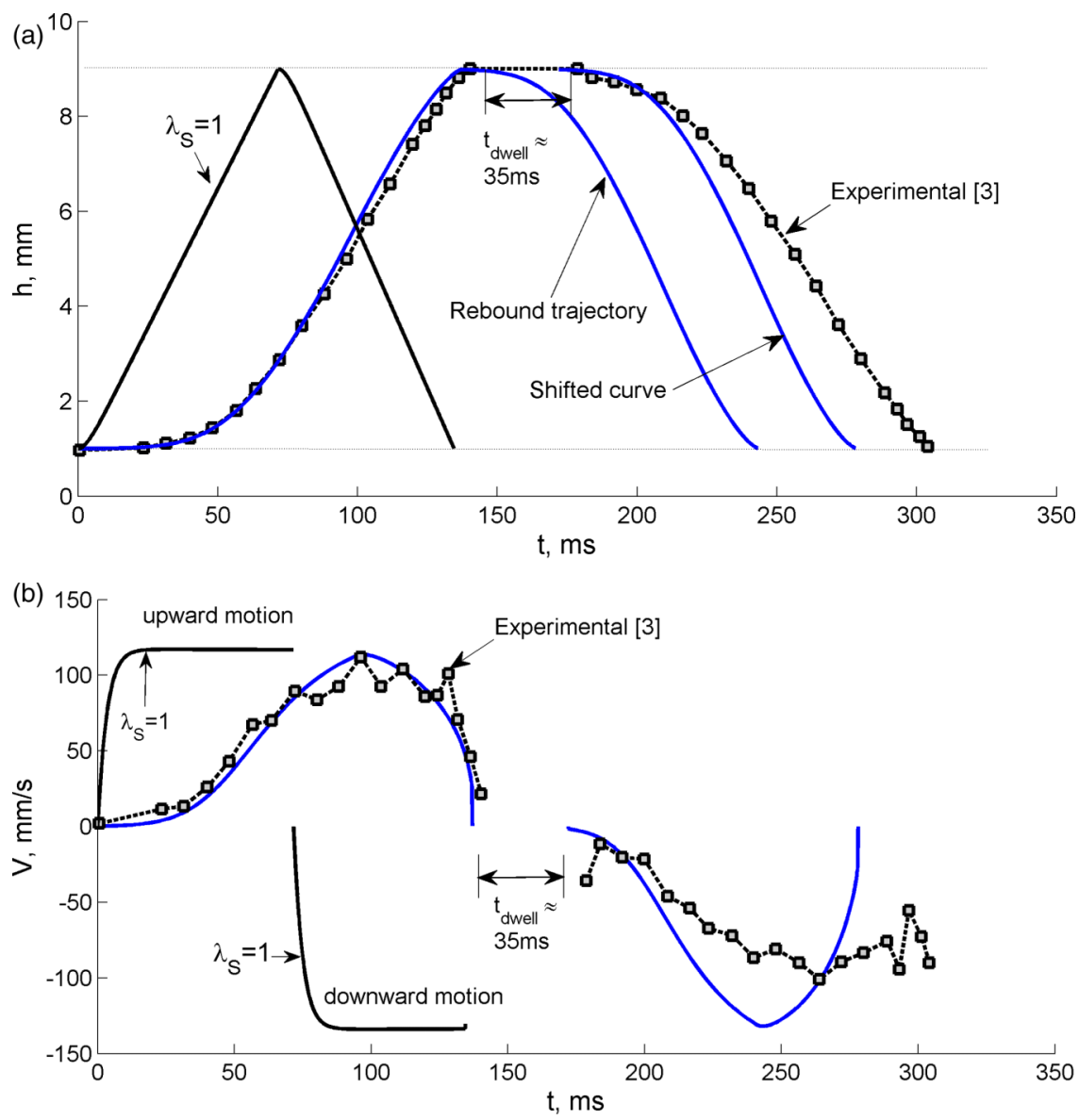

FIG. 15. Numerical and experimental data of the glassy carbon particle motion (case 1); temporal evolution of the particle trajectory (a) and the particle velocity (b). Numerical results were depicted by lines and obtained by using $s_{m d}=2 \mu \mathrm{m}$ $\left(\bar{S}_{m d}=2 \times 10^{-3}\right)$. 
which has been reported in Ref. 3. In fact, simplification of the particle equation of motion particularly, elimination of the wall effect, has caused such a significant error.

As seen in Figure 15, neglecting the wall effect $\left(\lambda_{S}=1\right)$ causes a very large error in predicting the temporal evolution of both the trajectory and the velocity of the particle. In contrast, when the wall effect is taken into account, the model results show good agreement with the experimental data. In particular, the upward motion is predicted very well when an approximate value of $2 \mu \mathrm{m}$ is considered for the microdischarge point $\left(\bar{S}_{m d}=2 \times 10^{-3}\right)$, which is a reasonable value. In contrast, the downward motion is not well predicted by the model. As reported in Ref. 3, the glassy carbon particle settles on the upper electrode for a while $\left(t_{d w e l l} \approx 35 \mathrm{~ms}\right)$ before detaching from it. The origin of this behavior is not clear yet. However, as seen in Figure 15(a) even after shifting the rebound trajectory from $t=135.7 \mathrm{~ms}$ to $t=135.7 \mathrm{~ms}+t_{d w e l l}$, again considerable discrepancy exists between the numerical prediction and the experimental data. Possible reasons are as follows: The most probable reason for such disagreement is the charge transfer between the glassy carbon particle and the upper electrode. Probably less charge is transferred to the particle during the "collision" with the upper electrode. Unfortunately, the charge exchange phenomenon in the configuration under study has not been well understood up to now, and as we mentioned earlier in Subsection VII A, there is a contradiction in the available literature in this regard and complementary investigation is required to find more details. Here, a natural question comes to mind: Is there any special amount of charge, $Q_{m d, 2}$, for which the proposed model predicts correctly the downward motion of the particle? $\left(Q_{m d, 2}\right.$ denotes for the particle initial charge at the instant of particle rebound.) The answer is positive. Using try and error technique, we found that the downward motion can be correctly predicted assuming $Q_{m d, 2}=0.81 Q_{t h}$ as seen in Figure 16. This approach has been applied previously in some models. For example, Bernanek et al. ${ }^{54}$ used the exchanged charge value as a free parameter to adopt their model predictions with the experimental data. Figure 16 indicates that the reduction of the particle charge in downward motion can significantly retard the particle downward trajectory. We shall refer to Figure 8 in order to find out the reason for this considerable retardation in the downward motion of the particle. As seen in Figure 8, when the particle is very close to the facing electrode (some microns), even a little reduction in the particle charge can result in large decrease in the repulsive electric force. Even attractive electric force can be achieved by extra reduction of the particle charge which can stick the particle to the electrode and prevent from falling. It seems that settling/dwelling time originates from the particle charging phenomenon.

In Figure 17, the numerical results of the present model are compared with the experimental data for a steel particle of $2 \mathrm{~mm}$ diameter (case 2 in Table II). The electric potential is $10 \mathrm{kV}$ (in contrast to $8 \mathrm{kV}$ in the case 1). Other parameters are the same as in case 2 and can be seen in Table II. Two series of experimental data are seen in the figure. One of them has been adopted from Ref. 3 (square symbols) and the other pertains to the present work (circle symbols). Three

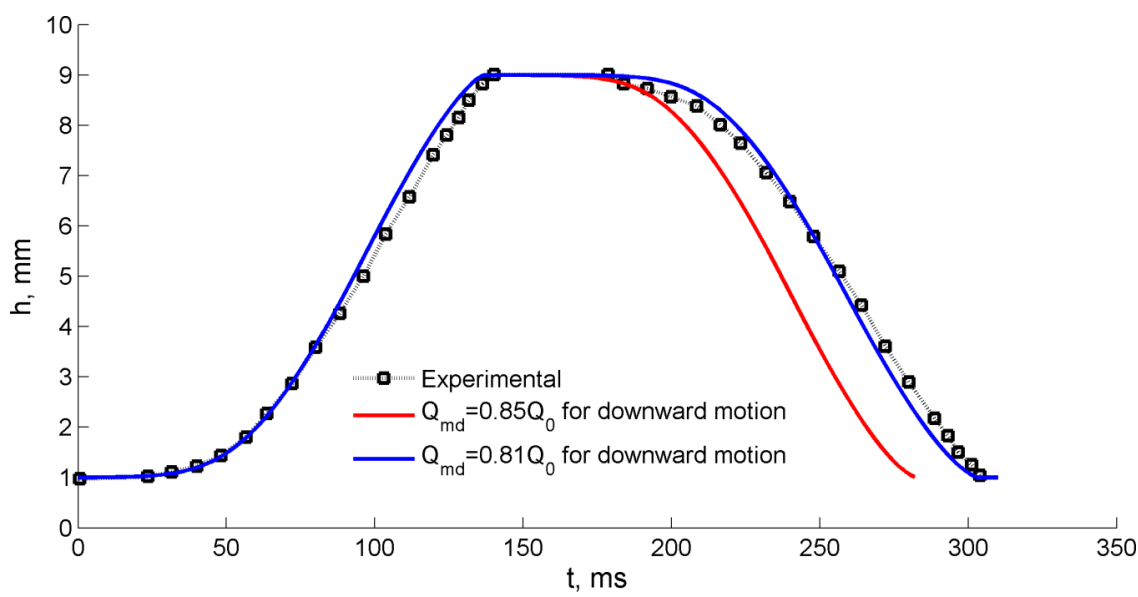

FIG. 16. Comparison between the experimental and predicted trajectory in which the dimensionless parameter $\Pi_{Q}$ has been considered as free parameter for the upper electrode. 


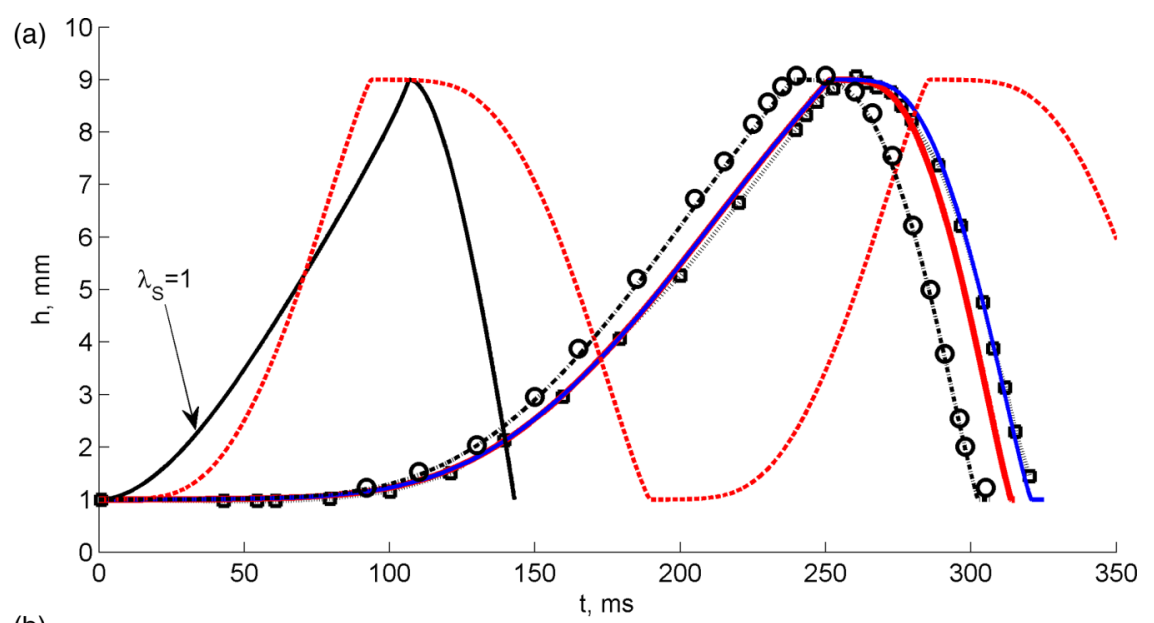

(b)

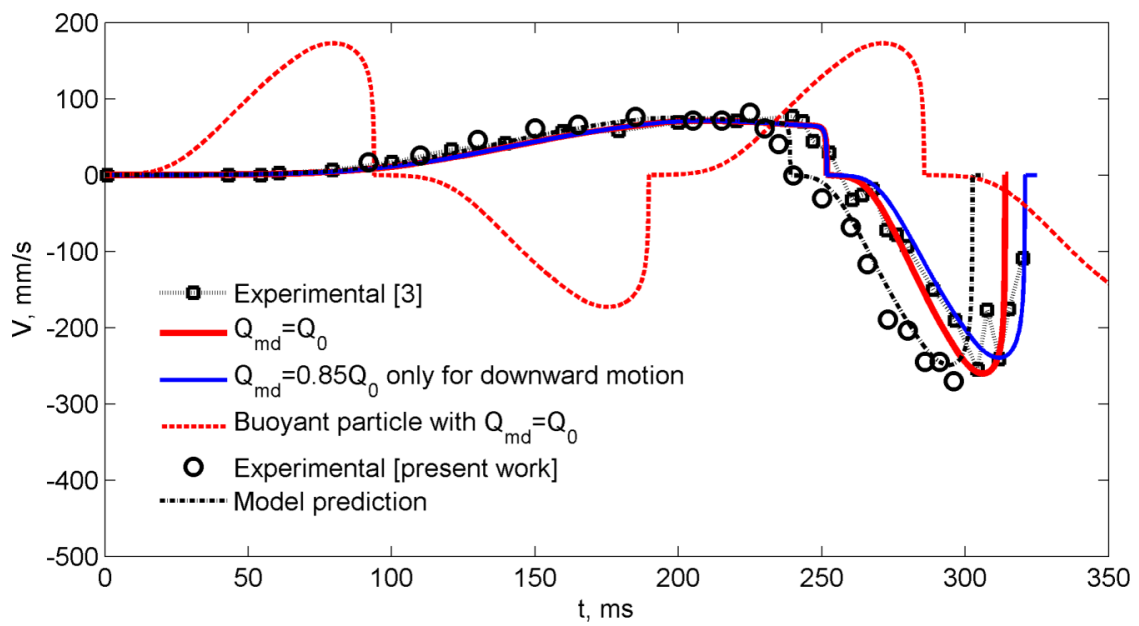

FIG. 17. Numerical and experimental data of the steel particle motion (case 2). Temporal evolution of the particle trajectory (a) and the particle velocity (b). The legend depicted in figure (b) holds for figure (a), as well. All the numerical results have been obtained using $s_{m d} \approx 1 \mu \mathrm{m}\left(\bar{S}_{m d} \approx 1 \times 10^{-3}\right)$. For better prediction of the experimental data, the model is run with $\Pi_{Q}=0.85$ for the downward motion of the submerged particle.

different frames showing the upward motion of the particle pertaining to the current study are shown in Figure 18. Present experiment has been carried out with the same condition as Ref. 3. However, as seen in Figure 17, there is a little difference between the two series of experimental data. Most probably, this is due to a little difference in the viscosity of silicon oil $(100 \mathrm{cS})$ which can occurr due to a limited difference in temperature of the two laboratory. Such estimation is confirmed by the model because the model results in a good prediction when it is run with a $5 \%$ lesser viscosity (see dashed-dotted line in Figures 17(a) and 17(b)). Generally, the results in Figure 17 are similar to the previous case. The position-time function is incorrectly predicted without taking the wall hydrodynamic effect into account (see Figure 17(a)). Very good agreement between the model numerical results and the experimental data for the upward motion is observed implying $Q_{m d}=Q_{t h}\left(\Pi_{Q}=1\right)$. However, a little faster downward motion is predicted for $Q_{m d}=Q_{t h}$. Again, and similar to the case 1 , the particle-electrode charge exchange is responsible for such discrepancy. This minor defect is improved by applying $Q_{m d}=0.85 Q_{t h}$ for the downward motion $(V<0)$.

Also, in order to show the effect of the particle apparent weight, we plot the trajectory and velocity of an equivalent buoyant particle in Figure 17 (red dashed line). For this end, the solution code was run with $\vec{F}_{g}+\vec{F}_{b}=0$ (see Eq. (21)). In this case, there is no difference between the upward and downward motion, and the only driving force is the electric force. Therefore, a comparison between $E_{0} Q_{0}$ and $\left(m_{p}-m_{f}\right) g$ can produce a scale to determine the possible difference between the 

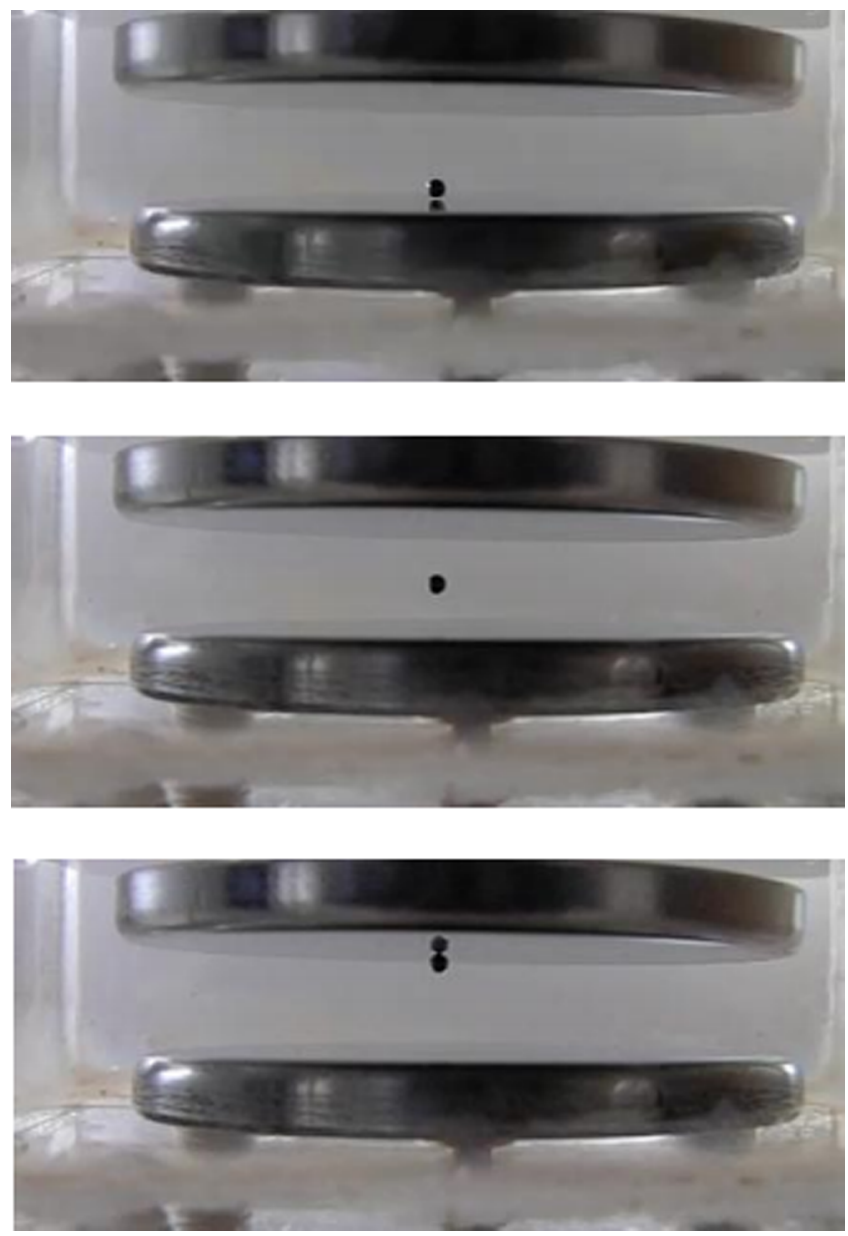

FIG. 18. Three different frames of the steel particle motion. The particle diameter and the gap between the two parallel electrodes are $2 \mathrm{~mm}$ and $10 \mathrm{~mm}$, respectively. The parallel electrode system and the particle submerged in silicon oil $(100 \mathrm{cS})$.

upward and downward motion of a typical particle. The apparent weight to the electric force ratio is $2\left(\rho_{p}-\rho_{f}\right) g R / \pi^{2} \varepsilon E_{0}^{2}$ which is exactly the same as $\Pi_{g}$ (see Eq. (28)). In Figure 19, period ratio of the downward-to-upward half-cycles, $T_{d} / T_{u}$, is depicted as a function of $\Pi_{g}$. It is clear that as $\Pi_{g} \rightarrow 1$, more difference appears between the upward and downward half-cycles periods in the particle trajectory. The model predictions are in good agreement with the experimental data as seen in Figure 15.

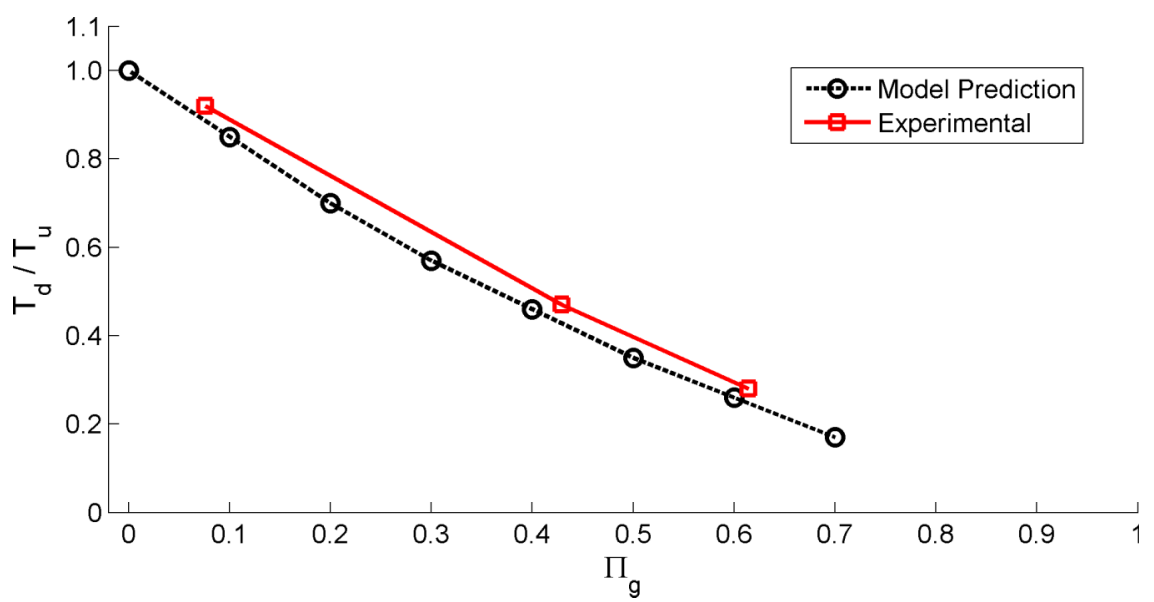

FIG. 19. The period ratio of the downward-to-upward half-cycles as a function of apparent weight to the electric force ratio. 
In spite of very good prediction shown in Figures 15-17, it is important to note that the history force has been ignored in the model. It is known that the history force makes a delay in the accelerated motion of a particle. ${ }^{31}$ Also, higher acceleration causes longer delay time. Although, oftentimes the history force can be ignored without anxiety about the accuracy of the results, it is important to note that in predicting the particle rebound trajectory, it might be important mainly due to its old wake. ${ }^{55}$ This has been confirmed by some studies. For example, Gondret et al. ${ }^{33}$ conducted an experimental study on the bouncing motion of solid spheres onto a solid plate. They compared some experimental data with numerical results and concluded that it is essential to take into account the history force in prediction of the bouncing trajectory for Reynolds number up to $10^{3}$. Rostamei et al. ${ }^{56}$ extended Gondret's study in a similar way to higher Reynolds number and found out similar result. They remarked that in prediction of the particle motion, from the starting point of motion to the wall impact, the history force effect is small (about 1\%-4.3\%) and can be ignored in comparison with the other hydrodynamic forces. But it significantly influences the bouncing trajectory of the particle after the first collision, even for the Reynolds numbers as much as 5000. Based on these findings, it deserves to investigate the history force effect on the particle trajectory in the configuration under study. We will focus on this issue in our next work.

\section{Effect of ionic conductivity of the working liquid}

It is worthy of particular attention that the conductivity of the surrounding liquid would strongly affect the particle dynamic behavior, since it determines the charge relaxation time and, subsequently, the Coulomb force. Therefore, it is very important to have a sufficiently accurate value of the liquid conductivity for the correct prediction of the particle velocity and trajectory. On the other hand, the conductivity of dielectric liquids would be affected by several agents including chemical impurities, water content, temperature, strengths of the applied electric field, and even the surface characteristics of the measuring electrodes. ${ }^{22,57}$ For instance, a very small amount of impurities would increase the conductivity of a dielectric liquid over hundred times. So, it is desirable to measure the liquid conductivity in the same conditions of the test. For this reason, the ionic conductivity was measured by two different methods including IRLAB system (model LDTRP-2) and directly using the two parallel electrode system of our test cell. The measuring procedure in our test cell was as follows. First, the plastic box and the electrodes were washed with cleaning liquid and dried properly. Then, an adequate amount of the sample liquid was poured into the plastic box. After adjusting the gap between electrodes, a DC voltage of a given value $\varphi$ was applied on the upper electrode and the consequent electric current, $I$, was recorded by the multimeter after almost 2 minutes (steady state). The conductivity was calculated by $\sigma=(A / d)(\varphi / I)$ in which $A$ is surface area of the electrode and other parameters are as before. There was no significant difference between the values given by IRLAB and the one measured by our system for low values of the applied voltage. However, the electric current showed deviations from the Ohmic behavior for voltages high enough. For the computations, we used the value of the liquid conductivity obtained using the parallel electrode system.

A series of experiments were conducted in order to examine the effect of fluid ionic conductivity on the dynamic behavior of the particle. All parameters pertaining to these experiments have been given in Table II (case $3 \mathrm{~A}, \mathrm{~B}$, and C). We used a small amount of butyl-alcohol as an additive to increase silicon oil conductivity. The results are depicted in Figure 20. First of all, it is observed that there is a very good agreement between the experimental data and the results obtained by the model. Such agreement demonstrates that the model has much predictive value. Note that in Figure 20(a) for each time $t$, the reported corresponding experimental data, $h$, have been derived by an arithmetic averaging of several data points (usually more than five). As seen in Figure 20(a), when the surrounding liquid is pure silicon oil with a conductivity of $\sigma=2.63 E-12 \mathrm{~S} / \mathrm{m}$ (case $3 \mathrm{~A})$, the particle keeps its upward motion until it collides with the upper electrode and then rebounds downwards. In this case the charge relaxation time is $\tau_{c}=8.4 \mathrm{~s}$, which is sufficiently larger than the upward motion time $\left(\theta_{c}=0.125\right)$. So the charge leakage does not modify the electric force during the particle motion. However, by adding $0.25 \mathrm{wt}$. \% butyl-alcohol into the pure sample oil, its conductivity increases nearly five times $(\sigma=5.22 E-11 \mathrm{~S} / \mathrm{m})$ which leads to $\tau_{c}=1.7 \mathrm{~s}$ (case 3-B). 

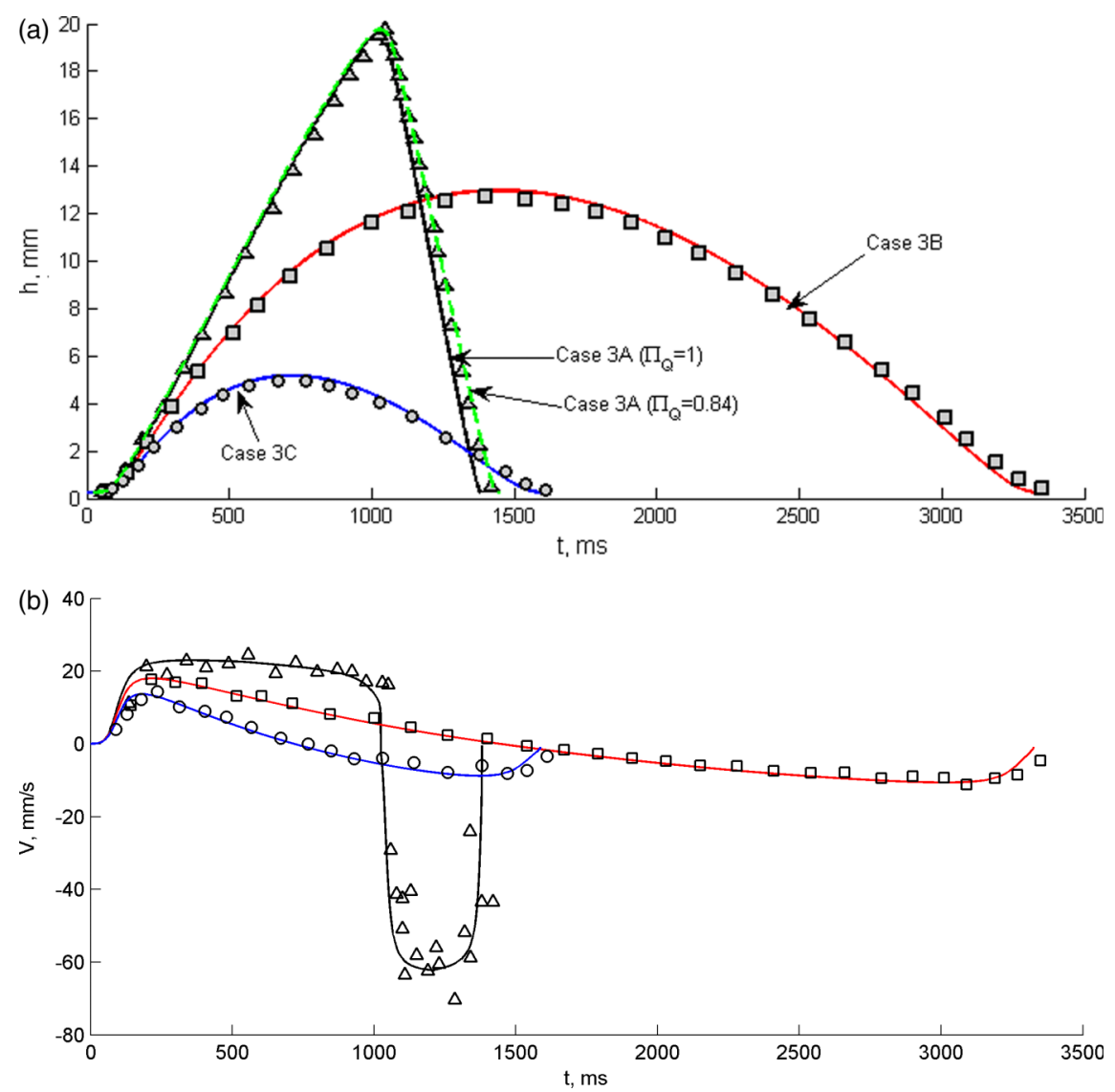

FIG. 20. Effect of liquid conductivity on the particle dynamics. (a) Temporal evolution of the particle trajectory, (b) velocity of the particle. Lines correspond to the output of the current model and symbols show the experimental data. $\sigma=2.63 E-12 \mathrm{~S} / \mathrm{m}$ (black line), $\sigma=5.22 E-11 \mathrm{~S} / \mathrm{m}$ (red line), $\sigma=2.62 E-11 \mathrm{~S} / \mathrm{m}$ (blue line). Other parameters can be found in Table II, Cases 3A-3C.

In this case, $\theta_{c}=1.968$. Thus, considerable charge leakage takes place, which gradually weakens the Coulomb force. As a consequence, the particle cannot continue its upward motion and falls down from $h=12.97 \mathrm{~mm}$. In the next case (case 3), which contains more butyl-alcohol (0.5 wt. \%), the flight-to-charge relaxation time is $\theta_{c}=1.917$, which in turn results in a sooner falling of the particle. It turns back from $h=5.17 \mathrm{~mm}$. It is important to note that trajectory of the downward motion of the moving particle is better predicted using $Q_{m d}=0.84 Q_{t h}$ instead of $Q_{m d}=Q_{t h}$ (see dashed green line in Figure 20(a)). Therefore, it can be deduced that similar to the previous cases, the particle obtains a little less charge from the upper electrode.

The particle velocity versus its position is shown in Figure 20(b). Similar to the other cases (see Figures 15(b) and 17), a particle velocity reduction is observed near the electrodes. This effect is a consequence of the wall hydrodynamic effect on the approaching particle. The present model predictions are in good agreement with the experimental data. Note that the data dispersion in the velocity profile (Figure 20(b)) comes from the first order differentiation of the trajectory data and can be reduced by increasing the number of data.

From these data, it can be deduced that $\tau_{c}$ plays a very important role in the particle dynamic behavior, particularly whenever its value is of the same order of the particle flight time. Therefore, the presence of a tiny amount of impurities in the surrounding liquid, or even on the particle surface, would significantly change the particle behavior. This convinces us that precise measurements of the surrounding liquid conductivity are an essential part of any experimental study related to this field. 


\section{CONCLUSIONS}

A simple and effective model was built for predicting the trajectory of the up and down motion of a conductive spherical particle submerged in a dielectric liquid subjected to a charge-free and uniform DC electric field on a parallel electrode system. Both hydrodynamic and electric interactions between the moving particle and the electrodes were taken into account, assuming the Stokes flow regime. Furthermore, the microdischarge effect on the rebound trajectory of the particle was included in the model, by accounting the sudden change of the magnitude and instantaneous reverse of the orientation of the electric force immediately after the microdischarge event. Outputs of the model were compared with some experimental data available in the literature, as well as with some additional experimental data obtained through the present study. Generally, good agreement was observed between the model predictions and the experimental data. The limited discrepancies may be attributed to the value of the charge transferred to the moving particle by the microdischarge phenomenon and the so-called history force. It was found out that the wall hydrodynamic effect and the dielectric liquid ionic conductivity are the most important factors in the prediction of the particle trajectory. It was shown that how the particle apparent weight may influence its dynamic behavior via the charge exchange phenomenon and how the microdischarge phenomenon can change the particle rebound trajectory. A lower bound was derived for the charge transferred to the particle while rebounding from an electrode. It is found that the time and length scales of the post-microdischarge motion of a millimeter sized particle can be as small as microsecond and micrometer, respectively. Based on these very small scales, the post-microdischarge motion was introduced as a possible reason for discrepancy between the experimental and the theoretical amount of the particle charge. The model is able to predict the so-called settling/dwelling time phenomenon for the first time.

\section{ACKNOWLEDGMENTS}

The first author expresses deeply his appreciation to Pablo García-Sánchez from Seville University for his valuable assistance and remarkable cooperation during the course of this research.

${ }^{1}$ L. Dascalescu and M. Mihailescu, "Mathematical modelling of conductive particle movement in dc corona fields," J. Electrost. 30, 297 (1993)

${ }^{2}$ O. D. Velev, S. Gangwal, and D. N. Petsev, "Particle-localized AC and DC manipulation and electrokinetics," Ann. Rep. Sect. C: Phys. Chem. 105, 213 (2009).

${ }^{3}$ C. Choi, K. Yatsuzuka, and K. Asano, "Dynamic motion of a conductive particle in viscous fluid under DC electric field," IEEE Trans. Ind. Appl. 37, 785 (2001).

${ }^{4}$ K. Asano, C.-r. Choi, K. Yatsuzuka, and D.-C. Lee, Acceleration and Deceleration of a Conductive Particle Within Parallel Electrodes in Viscous Fluid (IEEE, 2002).

${ }^{5}$ E. Capria, "Electrostatic manipulation of piezoelectric fibres using a sharp probe electrode in a dielectric liquid: Analysis of the electrohydrodynamic phenomena," Ph.D. thesis, School of Applied Sciences, Cranfield University, United Kingdom, 2007.

${ }^{6}$ J. Simola and J. Tuoriniemi, "Time-of-flight spectrometer for measuring the viscosity of fluids by using charged microparticles," Phys. B: Conden. Matter 165, 121 (1990)

${ }^{7}$ M. Bologa, V. Pushkov, and A. Berkov, "Electric field induced heat transfer enhancement in a gas-solid suspension heat exchanger," Int. J. Heat Mass Trans. 28, 1245 (1985).

${ }^{8}$ R. V. Garić-Grulović, Ž. B. Grbavčić, and Z. L. Arsenijević, "Heat transfer and flow pattern in vertical liquid-solids flow," Powder Technol. 145, 163 (2004).

${ }^{9}$ A. Paris and J. K. Eaton, "Turbulence attenuation in a particle-laden channel flow," Technical Report No. TSD-137, Department of Mechanical Engineering, Stanford University, Stanford, CA, 2001.

${ }^{10}$ D. Murray, "Local enhancement of heat transfer in a particulate cross flow-I Heat transfer mechanisms," Int. J. Multiphase Flow 20, 493 (1994).

${ }^{11}$ M. Haque and S. Arajs, "Electroconvective heat transfer in a suspension of rod-like akageneite particle ( $\beta$-FeOOH)," Int. J. Heat Mass Trans. 42, 1617 (1999).

${ }^{12}$ C. Choi, K. Yatsuzuka, and K. Asano, The Behavior of Spherical Particle Under Uniform Electric Field in Silicone Oil (IEEE, 2000).

$13 \mathrm{~J} . \mathrm{Wu}, \mathrm{J}$. Li, and Z. Xu, "An improved model for computing the trajectories of conductive particles in roll-type electrostatic separator for recycling metals from WEEE,” J. Hazard. Mater. 167, 489 (2009).

${ }^{14}$ L. Dascalescu, S. Das, and U. Kumar, "Numerical simulation of conductive particle behaviour at the surface of a plate electrode affected by a DC corona field," J. Electrostat. 67, 167 (2009).

${ }^{15} \mathrm{X}$. Wang, Z. Wang, and J. Noakhes, Motion of Conductive Particles and the Effect on AC Breakdown Strengths of Esters (IEEE, 2011). 
${ }^{16}$ K. D. J. Karunanayake and Y. Hoshino, "Electrostatic force acting on conductive ball between electrodes," J. Electrostat. 68, 91 (2010).

${ }^{17}$ M. Jalaal, B. Khorshidi, and E. Esmaeilzadeh, "An experimental study on the motion, deformation and electrical charging of water drops falling in oil in the presence of high voltage DC electric field," Exp. Therm. Fluid Sci. 34, 1498 (2010).

${ }^{18}$ B. Khorshidi, M. Jalaal, E. Esmaeilzadeh, and F. Mohammadi, "Characteristics of deformation and electrical charging of large water drops immersed in an insulating liquid on the electrode surface," J. Colloid Interface Sci. 352, 211 (2010).

19 A. T. Pérez, "Charge and force on a conducting sphere between two parallel electrodes," J. Electrostat. 56, 199 (2002).

${ }^{20}$ L. Dascalescu, M. Mihailescu, and R. Tobazeon, "Modeling of conductive particle behavior in insulating fluids affected by DC electric fields," IEEE Trans. Ind. Appl. 34, 66 (1998).

${ }^{21}$ N. Felici, "Forces et charges de petits objets en contact avec une électrode affectée d'un champ électrique," Rev. Gen. Electr. 75, 1145 (1966).

${ }^{22}$ T. J. Williams, "The resolution of water-in-oil emulsions by the application of an external electric field," Ph.D. thesis, University of Southampton, 1989.

${ }^{23}$ A. Khayari and A. T. Perez, "Charge acquired by a spherical ball bouncing on an electrode: Comparison between theory and experiment," IEEE Trans. Dielectr. Electr. Insul. 9, 589 (2002).

${ }^{24}$ R. Bartnikas, "A comment concerning the rise times of partial discharge pulses," IEEE Trans. Dielectr. Electr. Insul. 12, 196 (2005).

${ }^{25}$ R. Sarathi, A. Giridhar, and K. Sethupathi, "Analysis of partial discharge activity by a conducting particle in liquid nitrogen under AC voltages adopting UHF technique," Cryogenics 50, 43 (2010).

${ }^{26}$ A. Khayari, F. García, and A. Castellanos, Dynamics and Deformation of a Drop in a DC Electric Field (IEEE, 2003).

${ }^{27}$ S. Birlasekaran and M. Darveniza, "Microdischarges from particles in transformer oil," IEEE Trans. Electr. Insul. 4, 162 (1976).

${ }^{28}$ A. M. Drews, M. Kowalik, and K. J. Bishop, "Charge and force on a conductive sphere between two parallel electrodes: A Stokesian dynamics approach,” J. Appl. Phys. 116, 074903 (2014).

${ }^{29}$ L. Dascalescu, A. Mizuno, R. Tobazéon, P. Atten, R. Morar, A. Iuga, M. Mihailescu, and A. Samuila, "Charges and forces on conductive particles in roll-type corona-electrostatic separators," IEEE Trans. Ind. Appl. 31, 947 (1995).

${ }^{30}$ L. Pasol, M. Martin, M. Ekiel-Jeżewska, E. Wajnryb, J. BŁawzdziewicz, and F. Feuillebois, "Motion of a sphere parallel to plane walls in a Poiseuille flow. Application to field-flow fractionation and hydrodynamic chromatography," Chem. Eng. Sci. 66, 4078 (2011).

${ }^{31}$ C. E. Brennen, Fundamentals of Multiphase Flow (Cambridge University Press, 2005).

${ }^{32}$ L. Wakaba and S. Balachandar, "History force on a sphere in a weak linear shear flow," Int. J. Multiphase Flow 31, 996 (2005).

${ }^{33}$ P. Gondret, M. Lance, and L. Petit, "Bouncing motion of spherical particles in fluids," Phys. Fluids 14, 643 (2002).

${ }^{34}$ S. Xu and A. Nadim, "Oscillatory counter-centrifugation," Phys. Fluids 28, 021302 (2016).

${ }^{35}$ L. Bergougnoux, G. Bouchet, D. Lopez, and E. Guazzelli, "The motion of solid spherical particles falling in a cellular flow field at low Stokes number," Phys. Fluids 26, 093302 (2014).

${ }^{36}$ F. Candelier, J. Angilella, and M. Souhar, "On the effect of the Boussinesq-Basset force on the radial migration of a Stokes particle in a vortex," Phys. Fluids 16, 1765 (2004).

${ }^{37}$ S. Olivieri, F. Picano, G. Sardina, D. Iudicone, and L. Brandt, "The effect of the Basset history force on particle clustering in homogeneous and isotropic turbulence," Phys. Fluids 26, 041704 (2014).

${ }^{38}$ J.-R. Lin, "Squeeze film characteristics between a sphere and a flat plate: Couple stress fluid model," Comput. Struct. 75, $73(2000)$.

${ }^{39}$ A. D. Maude, "End effects in a falling-sphere viscometer," Br. J. Appl. Phys. 12, 293 (1961).

${ }^{40} \mathrm{H}$. Brenner, "The slow motion of a sphere through a viscous fluid towards a plane surface," Chem. Eng. Sci. 16, 242 (1961).

${ }^{41}$ Z. Adamczyk, M. Adamczyk, and T. Van de Ven, "Resistance coefficient of a solid sphere approaching plane and curved boundaries," J. Colloid Interface Sci. 96, 204 (1983).

${ }^{42}$ M. Simcik and M. Ruzicka, "Added mass of dispersed particles by CFD: Further results," Chem. Eng. Sci. 97, 366 (2013).

${ }^{43}$ X. Li, M. L. Hunt, and T. Colonius, "A contact model for normal immersed collisions between a particle and a wall," J. Fluid Mech. 691, 123 (2012).

${ }^{44}$ G. Joseph, R. Zenit, M. Hunt, and A. Rosenwinkel, "Particle-wall collisions in a viscous fluid," J. Fluid Mech. 433, 329 (2001).

${ }^{45}$ E. Izard, T. Bonometti, and L. Lacaze, "Modelling the dynamics of a sphere approaching and bouncing on a wall in a viscous fluid," J. Fluid Mech. 747, 422 (2014).

${ }^{46}$ A. Ardekani, D. Joseph, D. Dunn-Rankin, and R. Rangel, "Particle-wall collision in a viscoelastic fluid," J. Fluid Mech. 633, 475 (2009).

${ }^{47}$ N. Lecoq, R. Anthore, B. Cichocki, P. Szymczak, and F. Feuillebois, "Drag force on a sphere moving towards a corrugated wall," J. Fluid Mech. 513, 247 (2004).

${ }^{48}$ E. Loth and A. Dorgan, "An equation of motion for particles of finite Reynolds number and size," Environ. Fluid Mech. 9, 187 (2009).

${ }^{49}$ R. Cox and H. Brenner, "The slow motion of a sphere through a viscous fluid towards a plane surface-II. Small gap widths, including inertial effects," Chem. Eng. Sci. 22, 1753 (1967).

${ }^{50}$ F. L. Yang, "Interaction law for a collision between two solid particles in a viscous liquid," Ph.D. thesis, California Institute of Technology, 2006.

${ }^{51}$ A. M. Drews, C. A. Cartier, and K. J. M. Bishop, "Contact charge electrophoresis: Experiment and theory,” Langmuir 31, 3808 (2015). 
${ }^{52}$ R. Tobazèon, Proceedings 12th ICDL, Rome, Italy, Report No. 96CH35981, IEEE, 1996, pp. 309-312.

${ }^{53}$ Z.-G. Feng and E. E. Michaelides, "Inclusion of heat transfer computations for particle laden flows," Phys. Fluids 20, 040604 (2008).

${ }^{54}$ P. Beranek, R. Flittner, V. Hrobar, P. Ethgen, and M. Pribyl, "Oscillatory motion of water droplets in kerosene above co-planar electrodes in microfluidic chips," AIP Adv. 4, 067103 (2014).

${ }^{55}$ C. J. Lawrence' and R. Mei, "Long-time behaviour of the drag on a body in impulsive motion," J. Fluid Mech. 283, 307-327 (1995).

${ }^{56}$ M. Rostami, A. Ardeshir, and G. Ahmadi, "Can the history force be neglected for the motion of particles a thigh subcritical Reynolds number range?,” IJE Trans. B: Appl. 19, 1 (2006).

57 J. K. Park, J. C. Ryu, W. K. Kim, and K. H. Kang, "Effect of electric field on electrical conductivity of dielectric liquids mixed with polar additives: DC conductivity," J. Phys. Chem. B 113, 12271 (2009). 